\title{
Diffuse radio emission in MACS J0025.4-1222: the effect of a major merger on bulk separation of ICM components
}

\author{
C. J. Riseley ${ }^{1,2,3}$, A. M. M. Scaife ${ }^{1}$, M. W. Wise ${ }^{4,5}$, and A. O. Clarke ${ }^{1}$ \\ 1 Jodrell Bank Centre for Astrophysics, Alan Turing Building, School of Physics and Astronomy, The University of Manchester, \\ Oxford Road, Manchester, M13 9PL, UK \\ 2 CSIRO Astronomy \& Space Science, 26 Dick Perry Avenue, Kensington, WA 6151, Australia \\ e-mail: chris.riseley@csiro.au \\ 3 School of Physics \& Astronomy, University of Southampton, Highfield, Southampton, SO17 1BJ, UK \\ ${ }^{4}$ Netherlands Institute for Radio Astronomy (ASTRON), Postbus 2, 7990 AA Dwingeloo, The Netherlands \\ 5 Astronomical Institute Anton Pannekoek, University of Amsterdam, Postbus 94249, 1090 GE Amsterdam, The Netherlands
}

Received 15 August 2016 / Accepted 2 November 2016

\begin{abstract}
Context. Mergers of galaxy clusters are among the most energetic events in the Universe. These events have significant impact on the intra-cluster medium, depositing vast amounts of energy, often in the form of shocks, as well as heavily influencing the properties of the constituent galaxy population. Many clusters have been shown to host large-scale diffuse radio emission, known variously as radio haloes and relics. These sources arise as a result of electron (re-)acceleration in cluster-scale magnetic fields, although the processes by which this occurs are still poorly understood.

Aims. We present new, deep radio observations of the high-redshift galaxy cluster MACS J0025.4-1222, taken with the Giant Metrewave Radio Telescope (GMRT) at $325 \mathrm{MHz}$, as well as a new analysis of all archival Chandra X-ray observations. We aim to investigate the potential of diffuse radio emission and categorise the radio population of this cluster, which has only been covered previously by shallow radio surveys.

Methods. We produced low-resolution maps of MACS J0025.4-1222 through a combination of $u v$-tapering and subtracting the compact source population. Radial surface brightness and mass profiles were derived from the Chandra data. We also derived a 2D map of the intracluster medium temperature.

Results. For the first time, two sources of diffuse radio emission are detected in MACS J0025.4-1222, on linear scales of several hundred kpc. Given the redshift of the cluster and the assumed cosmology, these sources appear to be consistent with established trends in power scaling relations for radio relics. The X-ray temperature map presents evidence of an asymmetric temperature profile and tentative identification of a temperature jump associated with one relic.

Conclusions. We classify the pair of diffuse radio sources in this cluster as a pair of radio relics, given their consistency with scaling relations, location toward the cluster outskirts, and the available X-ray data.
\end{abstract}

Key words. radio continuum: general - galaxies: clusters: individual: MACS J0025.4-1222 - X-rays: galaxies: clusters

\section{Introduction}

Clusters and super-clusters of galaxies comprise the largest gravitationally-bound structures in the Universe, following the profile of dark matter distribution left over from high redshift. In the hierarchical model of structure formation, these objects grow through a number of processes, from constant accretion of matter from the local environment, to periodic consumption of small groups of galaxies and violent merger events involving collisions with other galaxy clusters. During merger events, vast amounts of gravitational energy $\left(\sim 10^{64} \mathrm{erg}\right.$; e.g. Ferrari et al. 2008) are deposited into the intracluster medium (ICM) during the timescale necessary for clusters to cross $(\sim 1 \mathrm{Gyr}$; Brunetti \& Jones 2014).

The most massive clusters of galaxies have masses of the order of $10^{15} M_{\odot}$, of which typically $\sim 70-80 \%$ is dark matter, and the remaining baryonic matter is split between the hot ICM gas $(\sim 15-20 \%)$ and galaxies (a few \%). The ICM gas is hot $(T \sim$ $\left.10^{8} \mathrm{~K}\right)$ and sparse $\left(n_{\text {gas }} \sim 10^{-3} \mathrm{~cm}^{-3}\right)$ and known to be permeated by a large-scale magnetic field. Major merger events strongly disrupt the structure and properties of galaxy clusters. They are believed to both trigger and quench periods of star formation in cluster member galaxies (e.g. Ma et al. 2010) as well as causing both large-scale disruption and heating of the ICM.

In a small number of galaxy clusters, merger events have been shown to cause physical separation in the components of the ICM. In the Bullet Cluster (1E 0657-56) for example there is clear separation between the peaks of the dark matter and baryonic matter distribution (e.g. Clowe et al. 2006; Bradač et al. 2006). In terms of observational indicators, lensing effects strongly trace the total mass distribution (which is dominated by dark matter), X-ray emission from galaxy clusters traces the thermal gas component and radio emission traces the non-thermal components (magnetic fields and the cosmic ray population).

Energy is primarily dissipated into the ICM in the form of shocks, as well as bulk motion of the ICM (e.g. Brunetti \& Jones 2014). Historically, shocks have mainly been detected in galaxy 
clusters through observations at X-ray wavelengths, typically presenting as sharp jumps in the surface brightness, temperature and density of the ICM (see Brunetti \& Jones 2014, for a recent review). However, toward the cluster outskirts, where stronger shocks are expected, the X-ray flux density is significantly lower than in the cluster centre, and shocks are far more difficult to detect.

Radio relics are a class of large-scale diffuse radio source associated with merging clusters. Observational data is broadly consistent with an interpretation of the diffusive shock acceleration (DSA; e.g. Feretti et al. 2012; Brunetti \& Jones 2014) mechanism: radio relics are usually aligned perpendicular to the merger axis, toward the outskirts of the cluster, and they generally exhibit a high polarisation fraction (indicative of highlyordered magnetic fields) with suggestions that the magnetic field is aligned with the outward-propagating shock front.

The association between relics and shocks has been confirmed in a growing number of clusters, including the Bullet Cluster (Shimwell et al. 2015), "El Gordo" (Botteon et al. 2016b), Abell 115 (Botteon et al. 2016a), Abell 754 (Macario et al. 2011), Abell 3376 (Akamatsu et al. 2012b), and Abell 3667 (e.g. Finoguenov et al. 2010; Akamatsu et al. 2012a). Questions are emerging, however, regarding the nature of the connection between shocks and radio relics as some observations have shown that radio relics are not always co-located with X-ray shocks (e.g. Ogrean et al. 2013). As such, clarifying the picture of the relationship between X-ray and radio properties of galaxy clusters is crucial to better understanding the processes by which these Mpc-scale sources of diffuse synchrotron emission come into being.

Many merging clusters have also been shown to radio haloes; amorphous, large-scale ( $\sim \mathrm{Mpc}$ size) radio sources associated with the ICM. Like relics, haloes are very diffuse (typically $\sim 0.1-1 \mu \mathrm{Jy} \operatorname{arcsec}^{-2}$ at $1.4 \mathrm{GHz}$ ). Unlike relics, however, these objects generally exhibit polarisation of less than a few per cent. Two exceptions to this are Abell 2255 (e.g. Pizzo et al. 2011) and MACS J0717.5+3745 (e.g. Bonafede et al. 2009) which both host haloes that exhibit a high degree of polarisation. However, this may be due to relics viewed in projection (in the case of MACS J0717.5+3745; see van Weeren et al. 2009) or highlypolarised filamentary structures within the halo (in the case of Abell 2255; see Govoni et al. 2005).

Two principal models of halo generation exist: turbulent acceleration models and secondary (or hadronic) models Within the turbulent acceleration framework (e.g. Brunetti et al 2001; Petrosian 2001), low-energy ( 1-10 GeV) electrons from the ICM are accelerated up to the radio emitting regime $(\gtrsim 10 \mathrm{GeV})$ by merger turbulence. Conversely, hadronic models (e.g. Dennison 1980; Blasi \& Colafrancesco 1999) suggest the electrons responsible for the observed synchrotron emission are injected into the ICM following inelastic collisions between relativistic protons and thermal ions.

The amorphous nature and low polarisation fraction of haloes are naturally explained within the context of the turbulent acceleration models, as the electrons responsible for the emission are located throughout the cluster ICM (explaining the typical similarity in extent between halo size and the X-ray emission) and the turbulence disrupts any large-scale ordering of magnetic fields (required for a high polarisation fraction). The large size of both relics and haloes requires efficient in-situ acceleration, as the lifetime of synchrotron-emitting electrons is significantly less than the typical timescale required for electrons to diffuse over the linear size of these objects (Jaffe 1977).
MACS J0025.4-1222 is a reasonably familiar massive cluster at high redshift. It is perhaps most well-known for being the second cluster where significant offset was found between the peaks in the dark matter and baryonic matter distributions (Bradač et al. 2008). This offset is interpreted as strong evidence for the low interaction cross-section of dark matter, as in the case of the Bullet cluster (e.g. Clowe et al. 2006; Bradač et al. 2006).

\subsection{MACS J0025.4-1222}

MACS J0025.4-1222 (hereafter MACS0025; J2000 right ascension and declination $\left.00^{\mathrm{h}} 25^{\mathrm{m}} 29.38^{\mathrm{s}}-12^{\circ} 22^{\prime} 37.0^{\prime \prime}\right)$ was identified in the Massive Cluster Survey (MACS; Ebeling et al. 2001) sample of high-luminosity, high-redshift clusters (Ebeling et al. 2007). MACS0025 is a double-cluster system believed to have undergone a recent major merger event. Based on a sample of 108 galaxies within $1.5 \mathrm{Mpc}$ of the cluster centre, Bradač et al. (2008) find the galaxy redshift distribution is consistent with a single Gaussian centred at a redshift of $z=0.5857$; the velocity dispersion is $\sigma_{\mathrm{cl}}=835_{-59}^{+58} \mathrm{~km} \mathrm{~s}^{-1}$. The redshift difference between the two sub-clusters is $\Delta z=0.0005 \pm 0.0004$; this indicates that the merger event is occurring close to the plane of the sky - within $5^{\circ}$ (Bradač et al. 2008).

The sub-clusters of MACS0025 have very similar total masses: for the north-west sub-cluster, the total mass is $M=$ $2.6_{-1.4}^{+0.5} \times 10^{14} M_{\odot}$; for the south-east sub-cluster the total mass is $M=2.5_{-1.7}^{+1.0} \times 10^{14} M_{\odot}$, measured within $300 \mathrm{kpc}$ of the brightest cluster galaxy (BCG) of each sub-cluster (Bradač et al. 2008). With such similar masses, theory suggests that a major merger should generate a pair of similar shocks propagating out from the cluster centre.

However, X-ray observations to-date have been unable to detect any significant shocks - Bradač et al. (2008) present 1-dimensional radial temperature measurements only. Additionally, MACS0025 is relatively unexplored at radio wavelengths: Paul et al. (2014) report the only published radio observations to-date. Their observations were conducted with the Giant Metrewave Radio Telescope (GMRT) at 610 and $235 \mathrm{MHz}$; no diffuse emission was detected, however the integration time of $5 \mathrm{~h}$ will have been a limiting factor when observing this highredshift cluster.

Ma et al. (2010) perform a deep study of the morphological, spectroscopic and photometric properties of galaxies within the MACS0025 region using the Hubble Space Telescope (HST) and Keck. Based on 436 galaxy spectra, they identify 212 cluster members within $4 \mathrm{Mpc}$ of the cluster centre. They find the global fraction of spiral and lenticular galaxies to be among the highest observed in high-redshift clusters. Additionally, they find six of the 212 cluster members to be post-starburst galaxies, all concentrated in the cluster centre, between the dark matter peaks. They propose that the starburst phase of these galaxies was both triggered and extinguished by the cluster merger, and that first core passage occurred 0.5-1 Gyr ago.

In this work we present a multi-wavelength study of MACS0025, based on new, deep radio observations with the GMRT and re-processed archival Chandra data. Throughout this work, we assume a concordance cosmology of $H_{0}=$ $73 \mathrm{~km} \mathrm{~s}^{-1} \mathrm{Mpc}^{-1}, \Omega_{\mathrm{m}}=0.27, \Omega_{\mathrm{vac}}=0.73$. All errors are quoted to $1 \sigma$. We adopt the spectral index convention that $S \propto v^{\alpha}$ and we take the redshift of MACS0025 to be 0.5857 (Bradač et al. 2008). At this redshift, an angular distance of $1^{\prime \prime}$ corresponds to $6.416 \mathrm{kpc}$ (Wright 2006). 
Table 1. Summary of the GMRT observations of MACS0025.

\begin{tabular}{|c|c|c|c|c|c|}
\hline Date & $\begin{array}{l}\text { Start time } \\
\quad \text { (IST) }\end{array}$ & Hours observed & \# Antennas & Antennas missing & Comments \\
\hline 2014 Jan. 12 & 13:00 & 8.25 & 29 & $\mathrm{C} 10$ & $\begin{array}{l}\text { S02 down for maintenance } 14: 50-16: 26 \text {. } \\
\text { C10 started observing at 18:09. C00-C03 no } \\
\text { fringes between 16:53 and 18:13. Reset at } \\
\text { 18:18 due to correlator problem. }\end{array}$ \\
\hline 2014 Jan. 13 & 18:09 & 4.00 & 28 & C10, W02 & C10 (W02) started observing at 18:35 (18:57). \\
\hline 2014 Feb. 01 & $15: 00$ & 6.00 & 28 & $\mathrm{C} 14, \mathrm{~W} 02$ & $\begin{array}{l}\text { Issues with power level settings forced reset at } \\
\text { 16:18, data taken beforehand were rejected. }\end{array}$ \\
\hline
\end{tabular}

\section{Observations and data reduction}

\subsection{GMRT data}

\subsubsection{Observations}

MACS0025 was observed with the GMRT for a total of three nights during January and February 2014, for a total of approximately $15 \mathrm{~h}$. Data were taken with the GMRT Software Backend (GSB; Roy et al. 2010) in $325 \mathrm{MHz}$ mode, with the default settings. Data were recorded every $8.05 \mathrm{~s}$, with an acquisition bandwidth of $33 \mathrm{MHz}$ and 256 channels. Two polarisation products were collected.

Throughout all observations, a minimum of 28 antennas were functional; any missing antennas were taken for painting or down due to problems with the hardware. Two major failures occurred during the observing runs; antenna fringe failures on 12th January resulted in a system reset and significant power issues occurred during the first hour of observing on 1st February. Compromised data were discarded for a total integration time on target of approximately $12 \mathrm{~h}$. A summary of each night's observations, including integration time, number of working antennas and any general comments are listed in Table 1.

On each night, the observations of the interleaved phase calibrator (0018-127; selected from the VLA calibrator manual ${ }^{1}$ ) and target (MACS0025) were bracketed at the beginning and end of the run by a 15-min scan on a primary calibrator source: either 3C 147, 3C 48 or 3C 468.1, depending on availability. The final $u v$-coverage of the GMRT observations of MACS0025 is shown in Fig. 1, where both the full $u v$-plane and a zoom on the short $(<5 \mathrm{k} \lambda)$ baselines is presented.

\subsubsection{Data reduction}

The data were reduced using the Source Peeling and Atmospheric Modelling (SPAM) software (Intema et al. 2009) which employs NRAO Astronomical Image Processing Software (AIPS) tasks through the ParselTongue interface (Kettenis et al. 2006). SPAM employs the Scaife \& Heald (2012; hereafter SH12) flux density scale, which provides models for a set of six calibrator sources designed to be used at low frequencies (particularly with LOFAR; van Haarlem et al. 2013). These sources all have flux densities that are stable over long periods, with well-understood spectral energy distributions (SED) and are compact compared to the resolution of LOFAR, to enable use of simple calibration models. In SH12, calibrators are selected from the 3C (Edge et al. 1959) and revised 3C (3CR; Bennett 1962) catalogues based on three criteria. Firstly, they must be at declination north of $\delta=20^{\circ}$. Secondly, they must have an

\footnotetext{
1 http://www.aoc.nrao.edu/ gtaylor/csource.html
}
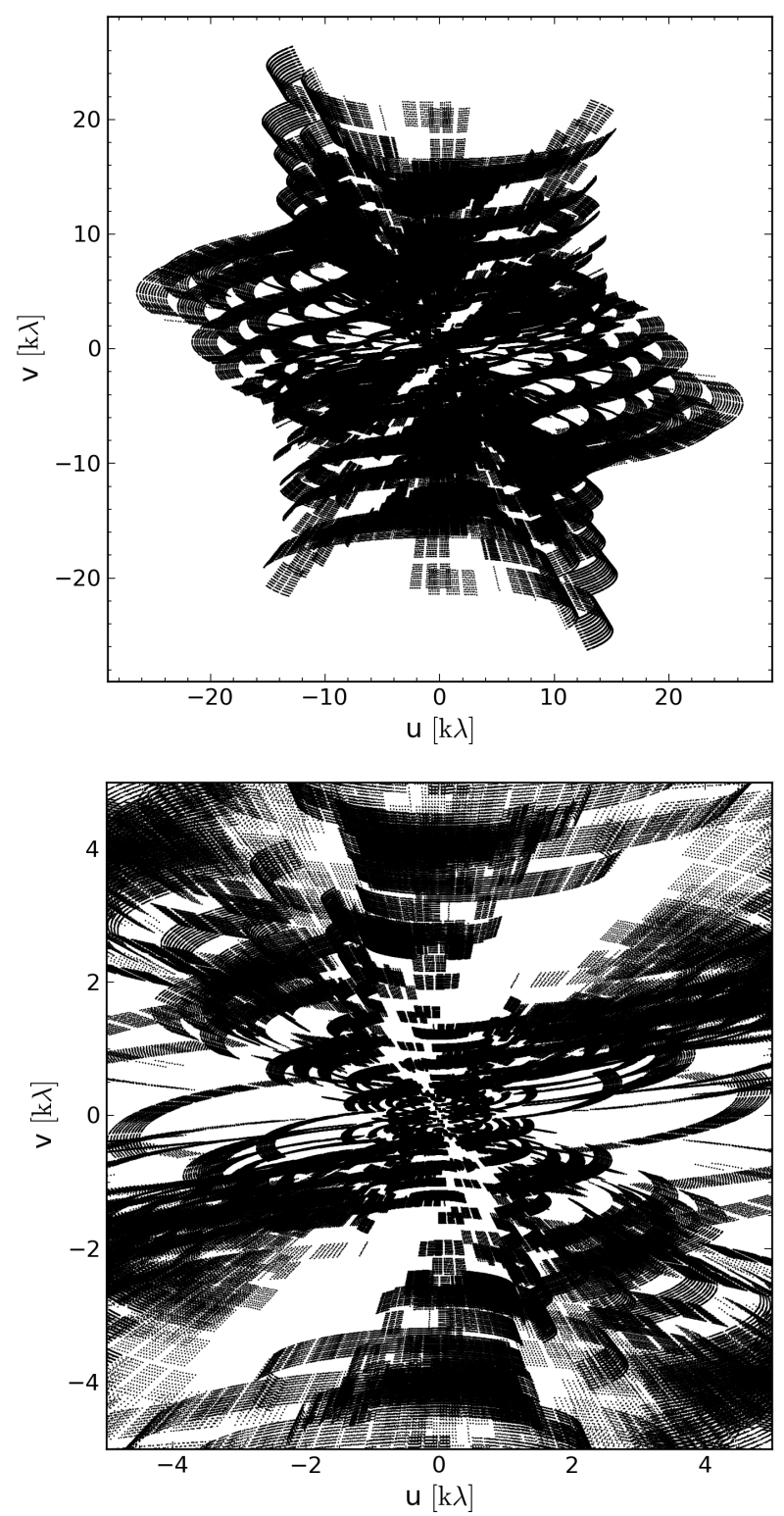

Fig. 1. $u v$-coverage of GMRT observations of MACS0025. Top: full $u v$ range. Bottom: zoom on the central portion of the $u v$-plane, illustrating the coverage on short baselines. Only one out of every ten data points are plotted.

integrated flux density greater than $20 \mathrm{Jy}$ at $178 \mathrm{MHz}$ and thirdly, they must have an angular diameter less than 20 arcsec. 
Table 2. Properties of calibrator sources used in this work, with flux density measurements and spectral index values on the Scaife \& Heald (2012) flux density scale.

\begin{tabular}{lcccc}
\hline \hline Name & $\begin{array}{c}\text { RA } \\
(\mathrm{J} 2000)\end{array}$ & $\begin{array}{c}\text { Dec } \\
(\mathrm{J} 2000)\end{array}$ & $\begin{array}{c}S_{325 \mathrm{MHz}} \\
{[\mathrm{Jy}]}\end{array}$ & $\alpha$ \\
\hline 3C 147 & $05^{\mathrm{h}} 42^{\mathrm{m}} 36.26^{\mathrm{s}}$ & $49^{\circ} 51^{\prime} 07.08^{\prime \prime}$ & 52.925 & -0.516 \\
3C 48 & $01^{\mathrm{h}} 37^{\mathrm{m}} 41.30^{\mathrm{s}}$ & $33^{\circ} 09^{\prime} 35.13^{\prime \prime}$ & 43.742 & -0.608 \\
3C 468.1 & $23^{\mathrm{h}} 50^{\mathrm{m}} 54.85^{\mathrm{s}}$ & $64^{\circ} 40^{\prime} 19.50^{\prime \prime}$ & 24.105 & -0.859 \\
\hline
\end{tabular}

The radio source $3 \mathrm{C} 468.1$ is available as a bandpass calibrator for the GMRT, although it is less well documented than 3C 48, 3C 147 and 3C 286. 3C 468.1 is also not on the SH12 flux scale. However, flux density measurements between $38 \mathrm{MHz}$ and 10.7 $\mathrm{GHz}$ are available from the literature, and in this work we use the measurements to extend the SH12 to include 3C 468.1 (see Appendix A).

Intema et al. (2009) describes data reduction with SPAM in detail; however here we will summarise the process. Following removal of edge channels, the data were averaged by a factor 4 in frequency (yielding 64 channels of width $502.8 \mathrm{kHz}$ ) and 2 in time, as a compromise between improving data processing speed and mitigating bandwidth-/time-smearing effects. Prior to calibration, the data were visually inspected for strong RFI and bad antennas/baselines. Calibration solutions were derived for the primary calibrators 3C 147, 3C 48 and 3C 468.1 and applied to the field, following standard techniques in SPAM. Properties of these calibrator sources are presented in Table 2.

The interleaved calibrator was not used during the reduction process - rather it was used to track data quality variation and atmospheric effects during the observing run itself. Instead, SPAM performs an initial phase calibration (and astrometry correction) using a sky model derived from the NRAO VLA Sky Survey (NVSS; Condon et al. 1998) before proceeding with three rounds of direction-independent phase-only self-calibration and imaging.

Following the self-calibration, SPAM identifies strong sources within the primary beam FWHM that are suitable for direction-dependent calibration and ionospheric correction. Only sources with well-defined astrometry are selected, in this case yielding a catalogue of approximately 20 sources. Subsequently, SPAM performs direction-dependent calibration on a per-facet basis, using the solutions to fit a global ionospheric model, as described by Intema et al. (2009). Throughout the reduction process, multiple automated flagging routines are used between cycles of imaging and self-calibration in order to reduce residual RFI and clip outliers. Overall, 55 per cent of the data were flagged; whilst this is high, this level of flagging is not uncommon for GMRT data at this frequency.

\subsubsection{Imaging}

During the self-calibration and imaging cycles, images were made using an AIPS ROBUST of -1.0 as a trade-off between sensitivity and resolution. Note that an AIPS ROBUST $=-5.0$ indicates pure uniform weighting, for maximum resolution; ROBUST $=+5.0$ indicates pure natural weighting, for maximum sensitivity. All imaging was performed with facet-based widefield imaging as implemented in SPAM. Three different images were produced following calibration: a full-resolution image including the full $u v$-range, with an AIPS ROBUST -1.0; a highresolution image made using baselines longer than $2 \mathrm{k} \lambda$, made to
Table 3. Observation log of Chandra data for MACS0025.

\begin{tabular}{lccr}
\hline \hline OBSID & Instrument & Date & Exposure $(\mathrm{ks})^{a}$ \\
\hline 3251 & ACIS-I & $2002-11-11$ & 18.9 \\
5010 & ACIS-I & $2004-08-09$ & 24.8 \\
10413 & ACIS-I & $2008-10-16$ & 75.1 \\
10786 & ACIS-I & $2008-10-18$ & 13.9 \\
10797 & ACIS-I & $2008-10-21$ & 23.9 \\
\hline Total & & & 156.6
\end{tabular}

Notes. ${ }^{(a)}$ Exposure times reflect the final exposure after screening for flares.

filter out emission on $\sim \mathrm{Mpc}$ scales; and a low-resolution image made after subtracting the clean-component model of the highresolution image, with a $u v$-taper of $5 \mathrm{k} \lambda$ to improve sensitivity to diffuse emission.

In Fig. 2, we present the entire field-of-view of the GMRT observations of MACS0025, imaged with an AIPS ROBUST of -1.0 . The MACS0025 field is rich, with many complex radio sources, including a number of radio galaxies (RG) hosting emission on a wide variety of scales. A number of these are highlighted in Fig. 2, and we discuss these sources further in Appendix B, where we present postage stamps of these sources (Fig. B.1) and mark any potential hosts identified in the literature. Given the locations of these sources and the redshifts of identified hosts, none of these are associated with MACS0025.

Additionally, there are two bright radio sources located reasonably far to the south of the field centre. These are identified as $\mathrm{S} 1$ and S2 in Fig. 2; in the literature they are known respectively as PKS 0023-13 and MRC 0023-12B. With integrated flux densities of $2.70 \pm 0.27$ and $0.94 \pm 0.09 \mathrm{Jy}$ for S1 and S2 respectively (measured using imfit in CASA 4.1), these sources caused severe artefacts in the field. While calibration with SPAM has significantly reduced these artefacts, our dynamic range is still limited. We measure an image noise of $81 \mu \mathrm{Jy}^{\text {beam }}{ }^{-1}$ toward the field centre, whereas the predicted thermal noise for this observation is approximately $20 \mu \mathrm{Jy}$ beam $^{-1}$.

\subsection{Chandra data}

\subsubsection{Observations}

MACS0025 has been observed a total of five times by Chandra with the Advanced CCD Imaging Spectrometer (ACIS; Garmire et al. 2003). All exposures were taken with the ACIS-I imaging configuration and therefore have uniform spectral properties. We have extracted and reprocessed all of this existing data for the subsequent analysis. Table 3 lists the relevant characteristics of the individual exposures.

\subsubsection{Data reduction}

The individual datasets were reprocessed using CIAO 4.6 and CALDB 4.6.1.1 to apply the latest gain and calibration corrections. Standard filtering was applied to the event files to remove bad grades and pixels. Each of the individual ObsIDs were also examined for the presence of strong background flares. None of the exposures showed evidence for appreciable flare events, however, so no additional filtering was required. These reprocessed event files were used in all subsequent imaging and spectral analyses. The final, combined exposure time after all filtering was $156.6 \mathrm{ks}$. 


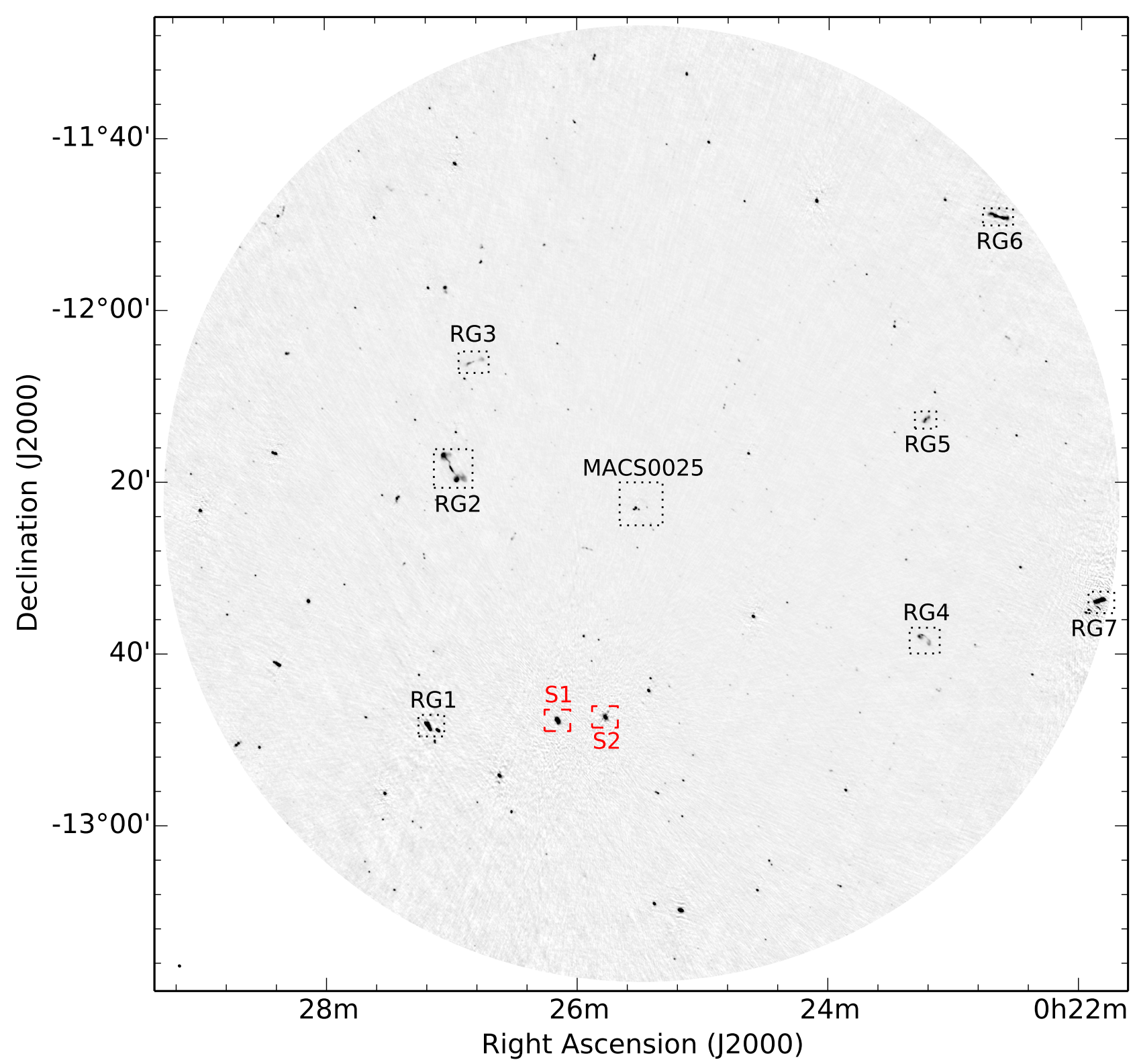

Fig. 2. Full field of view of the GMRT at $325 \mathrm{MHz}$ and at a resolution of $10.1 \times 8.3 \operatorname{arcsec}_{\text {. Image noise toward the field centre is } 81 \mu \mathrm{Jy} \text { beam }}{ }^{-1}$. Colour scale saturates at $10 \mathrm{mJy}_{\text {beam }}{ }^{-1}$ in order to emphasise emission from radio galaxies in the field. A number of the more extended radio galaxies are identified: these are further discussed in Appendix B. MACS0025 is also identified, as are the two brightest compact sources in the field, S1 and S2, which have integrated flux densities of $2.70 \pm 0.27$ and $0.94 \pm 0.09 \mathrm{Jy}$, respectively, at $325 \mathrm{MHz}$.

\subsubsection{Imaging}

A surface brightness mosaic of the field around MACS0025 was constructed by re-projecting the individual event files to a common tangent point on the sky and then combining them. To correct for sensitivity variations across the field, instrument and exposure maps were made for each ObsID individually and combined after re-projection to flat-field the resulting mosaic.

For the instrument maps, the spectral weighting was determined by fitting a single temperature, MEKAL thermal model (Mewe et al. 1985; Liedahl et al. 1995) plus foreground Galactic absorption to the total integrated spectrum from the central 90 arcsec region around MACS0025. The Galactic absorption was modelled as neutral gas with solar abundances and a column density fixed at $N_{\mathrm{H}}=2.38 \times 10^{20} \mathrm{~cm}^{-2}$ as determined by the LAB Survey of Galactic H I (Kalberla et al. 2005). This model gives a reasonable fit to the data with a reduced $\chi^{2}=1.04$ and best-fit temperature and metallicity of $k T=8.5 \mathrm{keV}$ and $Z=0.23$, respectively.

Individual background event files were created for each dataset from the standard ACIS blank-sky event files following the procedure described in Vikhlinin et al. (2005) and combined after re-projection to form a background mosaic. The resulting background-subtracted, exposure-corrected mosaic for the energy range $0.5-7.0 \mathrm{keV}$ is shown in Fig. 3.

\subsubsection{Spectral analysis}

We have performed spatially-resolved spectral analyses of the X-ray emission in MACS0025 considering both 1D and $2 \mathrm{D}$ adaptive binning. For the $2 \mathrm{D}$ analysis, the contour binning algorithm of Sanders (2006) was used, while for the 1D analysis a series of radially symmetric annuli were defined, centred on the 


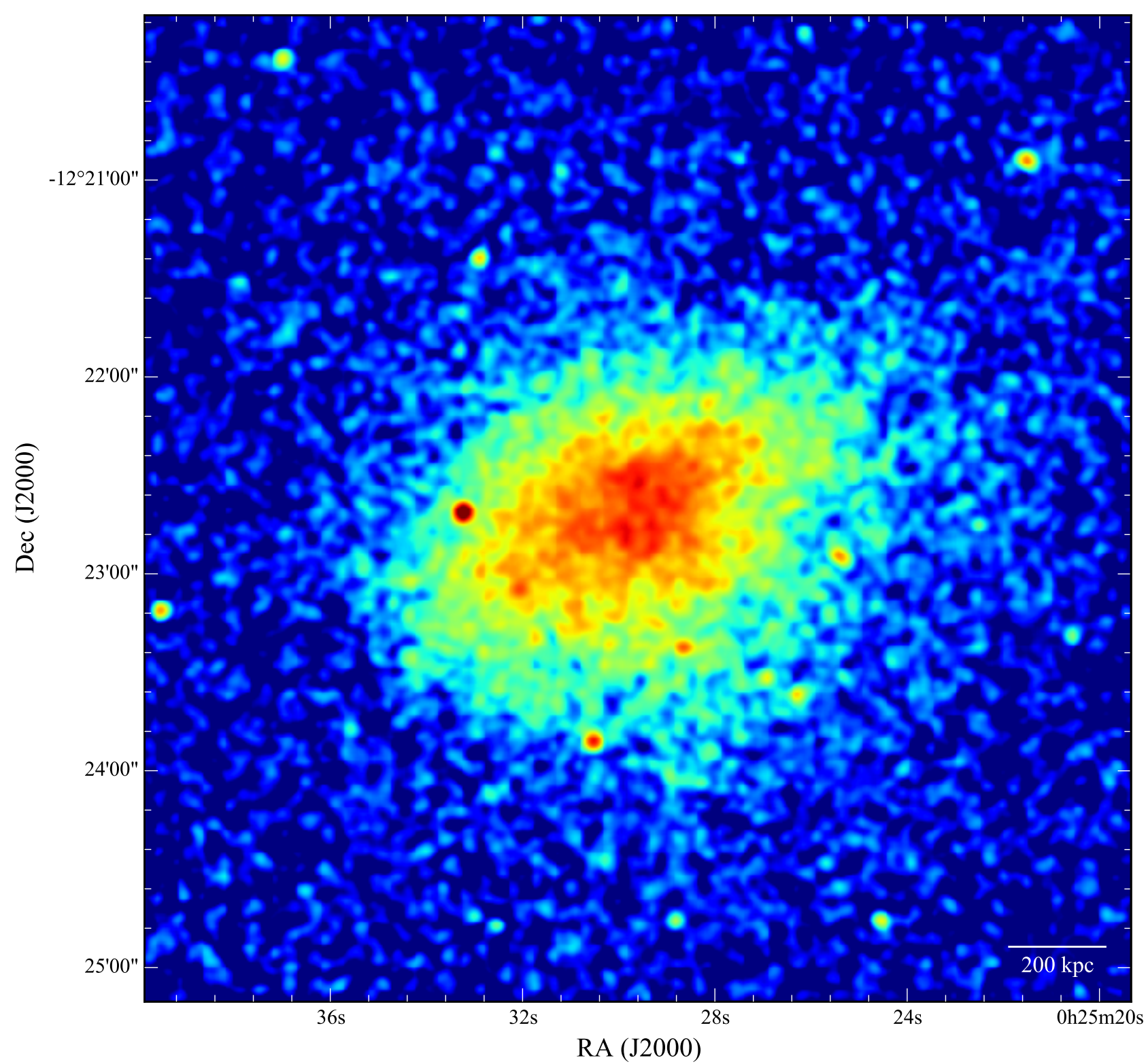

Fig. 3. Exposure-corrected, background-subtracted $0.5-7.0 \mathrm{keV}$ surface brightness mosaic for the central region around MACS0025 based on $156 \mathrm{ks}$ of existing Chandra archival data. The field measures $5 \times 5 \operatorname{arcmin}^{2}$ and has been smoothed with a $\sigma \sim 3^{\prime \prime}$ Gaussian.

peak of the X-ray flux density $\left(00^{\mathrm{h}} 25^{\mathrm{m}} 29.380^{\mathrm{s}}-12^{\circ} 22^{\prime} 37.06^{\prime \prime}\right)$. We note that due to the combination of the adaptive binning process and the signal-to-noise requirement on the extracted spectra, the definition of the resulting extraction regions are relatively insensitive to the exact choice of X-ray centroid. In both cases, all spectral extraction regions were defined adaptively so as to contain a specified signal-to-noise ratio after background subtraction. Within each region, source and background spectra, effective area, and response matrix files were created for each OBSID individually and then combined into a single, summed set of files for analysis using the CIAO tool combine_spectra. All subsequent spectral analysis was done using the Sherpa (Freeman et al. 2001) fitting package in CIAO.

\section{Results}

We present multi-wavelength images of MACS0025 in Fig. 4. Contours correspond to the full-resolution (AIPS ROBUST -1.0) radio data at $325 \mathrm{MHz}$ from the GMRT. In the top panel, the colour image is the X-ray surface brightness from archival
Chandra data as per Fig. 3 (details of which are shown in Table 3) displayed using the "cubehelix" colour scheme (Green 2011). The bottom panel presents the optical image of MACS0025 from HST data (proposal 10703, P.I. H. Ebeling) reduced using the default HST pipeline. The synthesised beam of the full-resolution GMRT image is $10.1 \times 8.3 \mathrm{arcsec}$; the measured off-source noise is $81 \mu \mathrm{Jy}_{\text {beam }}^{-1}$.

\subsection{Radio-optical cross identification}

From Fig. 4, it can be seen that we recover emission from multiple compact sources in the region of MACS0025, and that there is little indication of any diffuse emission at this resolution. The triplet of compact radio sources south-east of the cluster centre all appear to be associated with cluster-member galaxies, as identified by Bradač et al. (2008) and Ma et al. (2010). Figure 5 presents postage stamp images of the compact radio sources in MACS0025. 

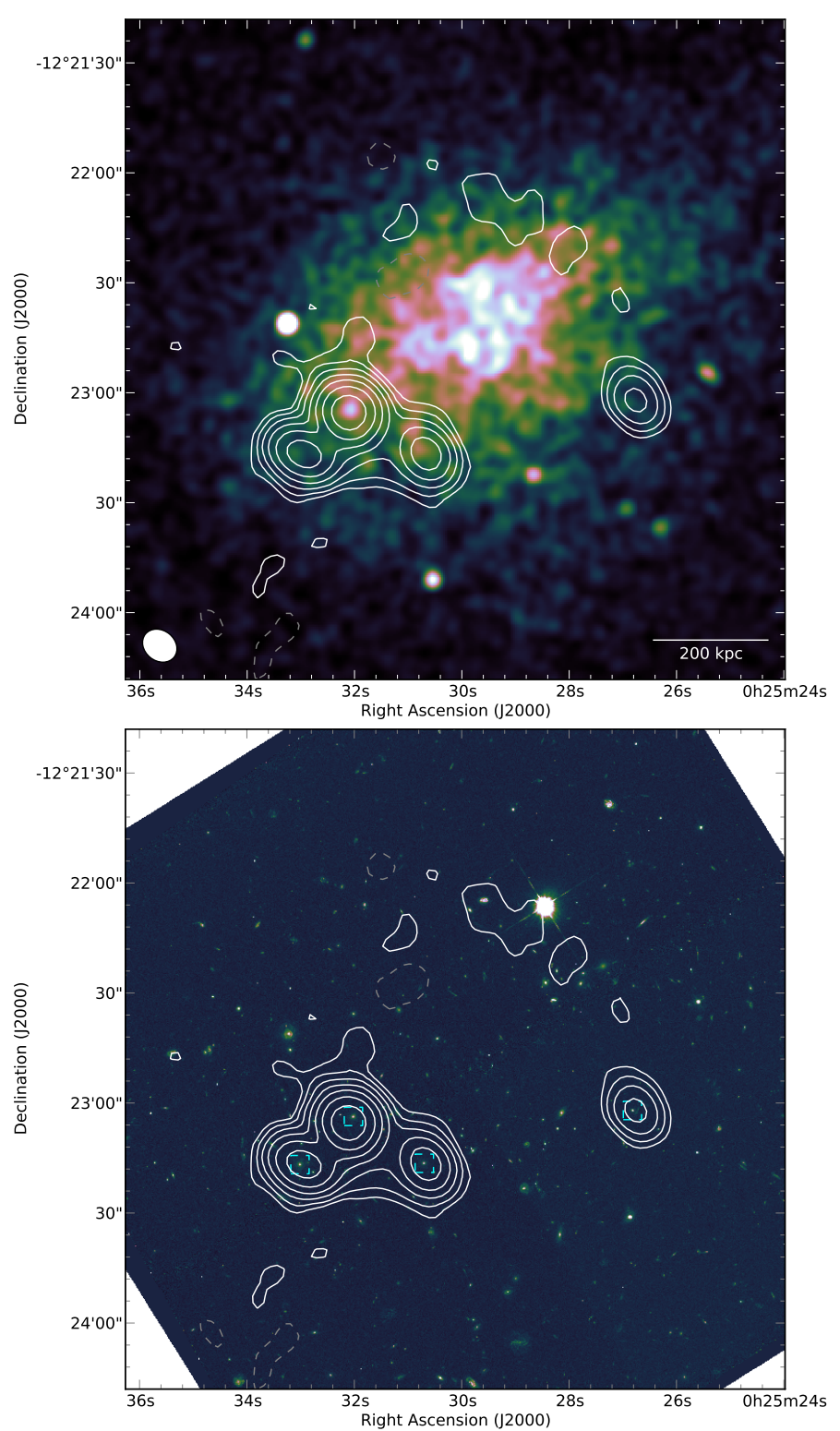

Fig. 4. Composite images of MACS0025. Top panel: colour image is $\mathrm{X}$-ray surface brightness as per Fig. 3. Bottom panel: colour image is HST image of the cluster centre (proposal 10703, P.I. H. Ebeling). Cyan boxes identify optical hosts for the compact radio sources. In both panels, contours are $325 \mathrm{MHz}$ GMRT data, imaged with an AIPS ROBUST -1.0 , at levels $[1,2,4,8,16,32,64] \times 5 \sigma_{\mathrm{FR}}$ where $\sigma_{\mathrm{FR}}=$ $81 \mu \mathrm{Jy}$ beam $^{-1}$. The $-5 \sigma$ contour is presented in gray. The synthesised beam is $10.1 \times 8.3 \operatorname{arcsec}$ at PA $51.1^{\circ}$, indicated by the unfilled ellipse in the lower-left corner.

The host galaxies of two of the compact radio sources to the SE of MACS0025 are the two BCGs in this sub-cluster (as identified by Bradač et al. 2008). These BCGs are identified in Fig. 5a. Additionally, from Fig. 4 the central radio source of the triplet appears to be associated with a faint X-ray point source, which may suggest a powerful AGN. Their optical spectra indicate that the hosts of these compact radio sources are all absorption-line elliptical galaxies (Ma et al. 2010).

\subsection{X-ray surface brightness profile}

The X-ray surface brightness distribution in MACS0025 shows a clear elliptical structure with the major axis of the ellipse

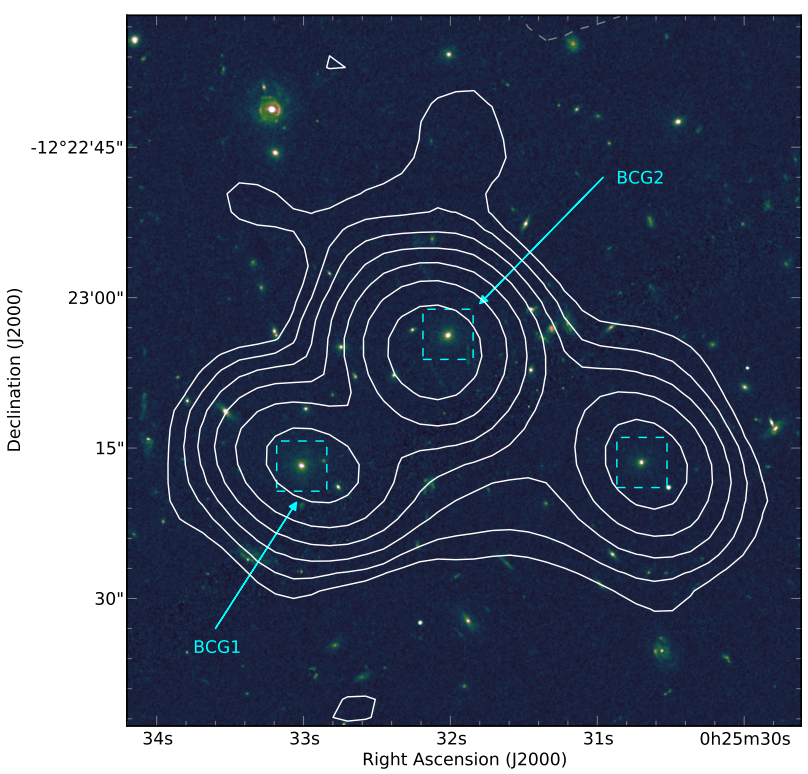

(a)

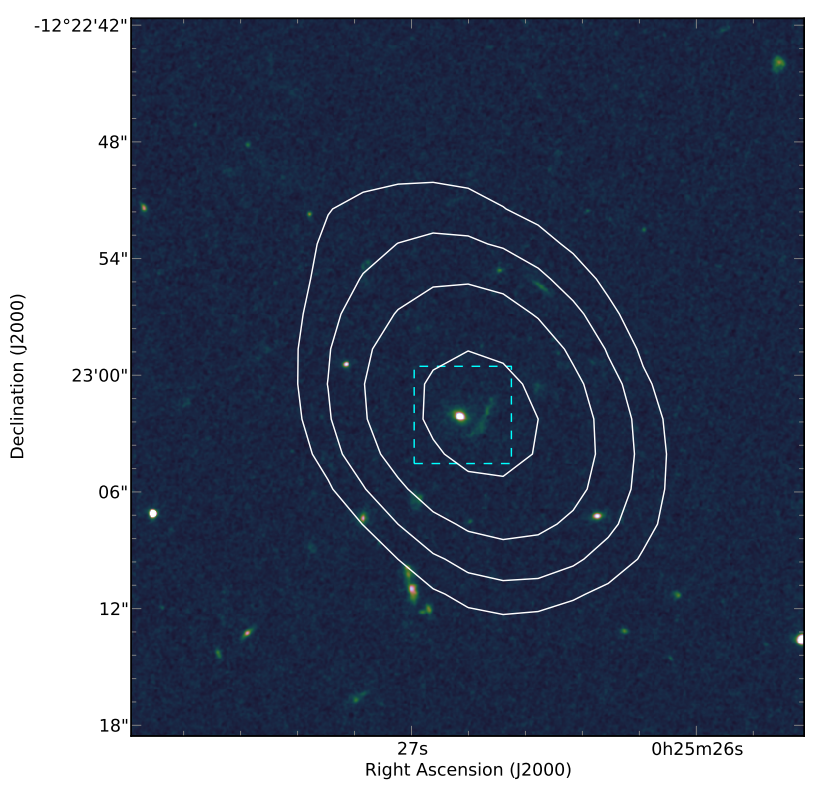

(b)

Fig. 5. Postage stamps of radio/optical associations for compact radio sources in MACS0025. Panel a) presents the triplet of compact radio sources in the SE sub-cluster of MACS0025, panel b) presents the single compact radio source in the NW sub-cluster. Contours are fullresolution GMRT data as per Fig. 4. The colour-scale is HST data as per Fig. 4. All selected optical hosts (cyan boxes) are cluster member galaxies, identified as absorption-line ellipticals by Ma et al. (2010).

oriented along what is presumed to be the merger axis in the system (see Fig. 3). A radial surface brightness profile was computed by defining a set of elliptical annuli centered on the peak of the X-ray emission. As discussed above, these annuli have been defined adaptively to include a minimum number of counts after background subtraction, thereby assuring a uniform signalto-noise ratio (SNR) in each bin. A fixed axial ratio of $b / a=2$ was assumed, where $b$ and $a$ are taken to be the major and minor axes of the elliptical distribution, respectively. The position angle (PA) of the X-ray emission was taken to be $120^{\circ}$ as measured counter-clockwise from north. 


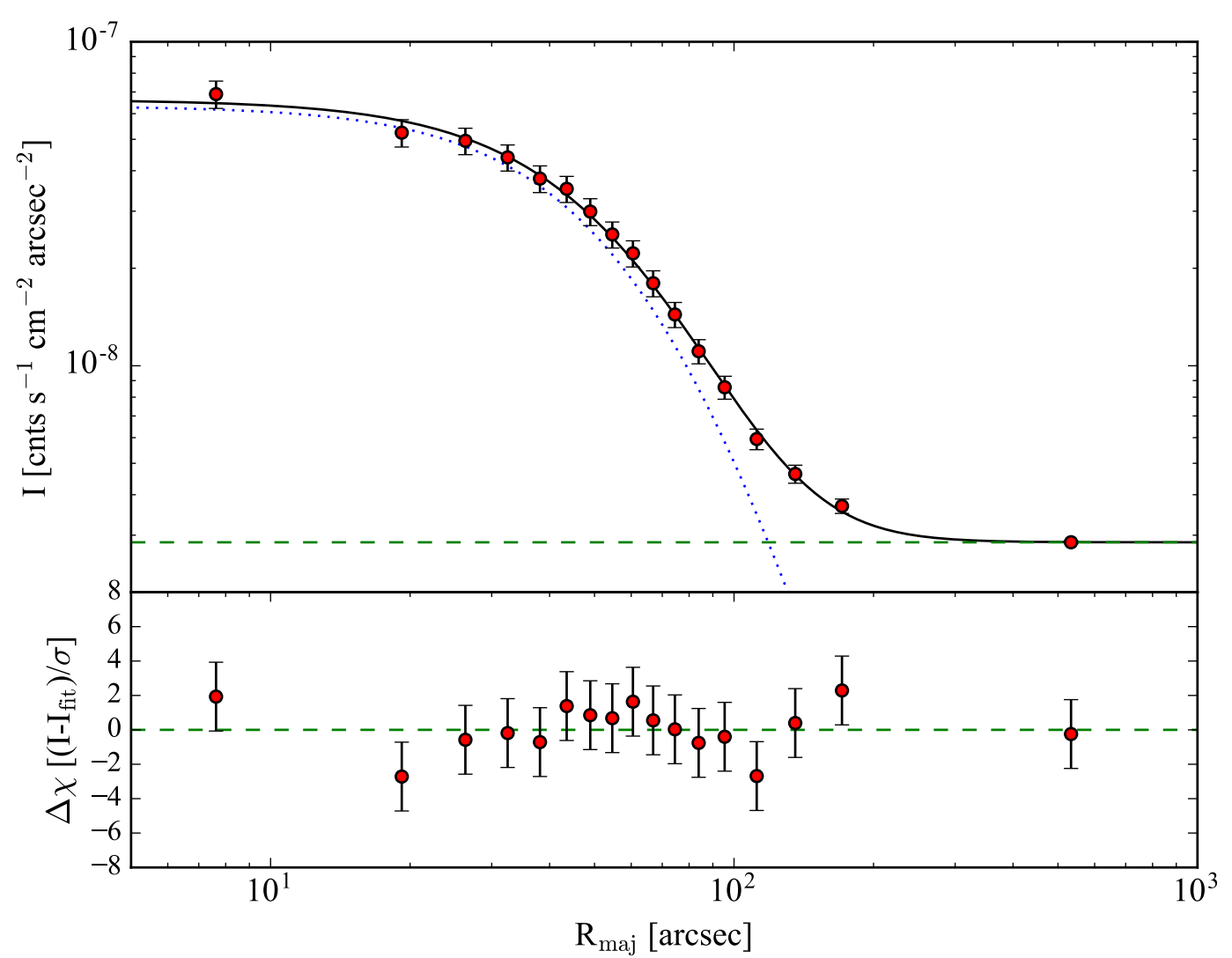

Fig. 6. Extracted surface brightness profile for MACS0025. In the upper panel, the solid line shows the best-fit model to the overall surface brightness. The background level is denoted by the horizontal, dashed line while the dotted curve indicates the underlying, background-subtracted model. The lower panel indicates the difference between data and model relative to the error at each radius. For both panels, the radius, $R_{\text {maj }}$, corresponds to the radial distance along the semi-major axis of the assumed elliptical distribution. The model is discussed in the text.

The resulting elliptical surface brightness profile is shown in Fig. 6. An isothermal $\beta$ profile (Cavaliere \& Fusco-Femiano 1976; Sarazin \& Bahcall 1977) of the form:

$I(r)=I_{0}\left(1+\frac{r^{2}}{r_{\mathrm{c}}^{2}}\right)^{-3 \beta+\frac{1}{2}}+I_{\mathrm{B}}$

provides a reasonable representation of the azimuthally averaged surface brightness profile where $I_{0}, r_{\mathrm{c}}$ and $\beta$ are the peak central surface brightness, core radius and exponential fall-off at large radii, respectively. $I_{\mathrm{B}}$ is a constant representing the contribution of the background. Given this model, we find best-fit values of $I_{0}=6.42 \pm 0.14 \times 10^{-8}$ cnts s$^{-1} \mathrm{~cm}^{-2}, r_{\mathrm{c}}=72^{\prime \prime} \pm 3^{\prime \prime}$ and $\beta=$ $0.94 \pm 0.04$ for these parameters. The corresponding best-fit value for the background surface brightness is $I_{\mathrm{B}}=2.85 \pm 0.01 \times$ $10^{-9} \mathrm{cnts} \mathrm{s}^{-1} \mathrm{~cm}^{-2}$.

\subsection{Mass profiles}

As we discuss in Sect. 4.1.2, de Gasperin et al. (2014) find evidence for a correlation between the observed $1.4 \mathrm{GHz}$ radio power for large-scale radio relics observed in clusters and the mass of the host cluster as inferred from SZ measurements with Planck (Planck Collaboration XXIX 2014). For an assumed spectral index, we can, in principle, use the GMRT observations presented here to assess whether the observed power of the radio relics in the MACS0025 cluster is consistent with this correlation. Unlike the sample presented in de Gasperin et al. (2014), however, the MACS0025 cluster was not detected by Planck, so no SZ-derived mass estimates are available. Here, we have instead utilized the observed X-ray properties to derive a mass for the cluster, under the assumption of hydrostatic equilibrium.
Given radial profiles for the gas properties in MACS0025, we can derive both the gas mass and total gravitating mass in the MACS0025 system. Assuming hydrostatic equilibrium and spherical symmetry, the total cluster mass, $M$, contained with a radius $r$ is given by

$M(<r)=-\frac{k T(r) r^{2}}{\mu m_{\mathrm{p}} G}\left(\frac{1}{n_{\mathrm{e}}} \frac{\mathrm{d} n_{\mathrm{e}}}{\mathrm{d} r}+\frac{1}{T} \frac{\mathrm{d} T}{\mathrm{~d} r}\right)$

where $T(r)$ and $n_{\mathrm{e}}(r)$ are the gas temperature and electron number density at radius $r$, respectively (Fabricant et al. 1980). Similarly, the gas mass can be calculated directly using

$M_{\text {gas }}(<r)=\mu m_{\mathrm{p}} \int n_{\mathrm{e}}(r) \mathrm{d} V$

where $\mathrm{d} V$ is the volume of the shell containing gas at density $n_{\mathrm{e}}(r)$, and $\mu m_{\mathrm{p}}$ is the mean mass per particle $1.0 \times 10^{-24} \mathrm{~g}$.

In order to determine $k T$ and $n_{\mathrm{e}}$ as a function of position in the cluster, we have extracted spectra for a series of elliptical annuli centered on the peak of the X-ray emission for the ellipsoidal geometry described in Sect. 3.2. We have used a SNR criteria of 40 which results in $\sim 1600$ net counts per bin after background subtraction in each region. Within each extraction region, a single temperature, MEKAL thermal model (Mewe et al. 1985; Liedahl et al. 1995) was fitted to the combined spectrum from that region. The absorption was set to the nominal Galactic value of $N_{\mathrm{H}}=2.38 \times 10^{20} \mathrm{~cm}^{-2}$ and the abundance was fixed to a value of $Z=0.23$ based on the fit to the mean spectrum as discussed in Sect. 2.2.3. 


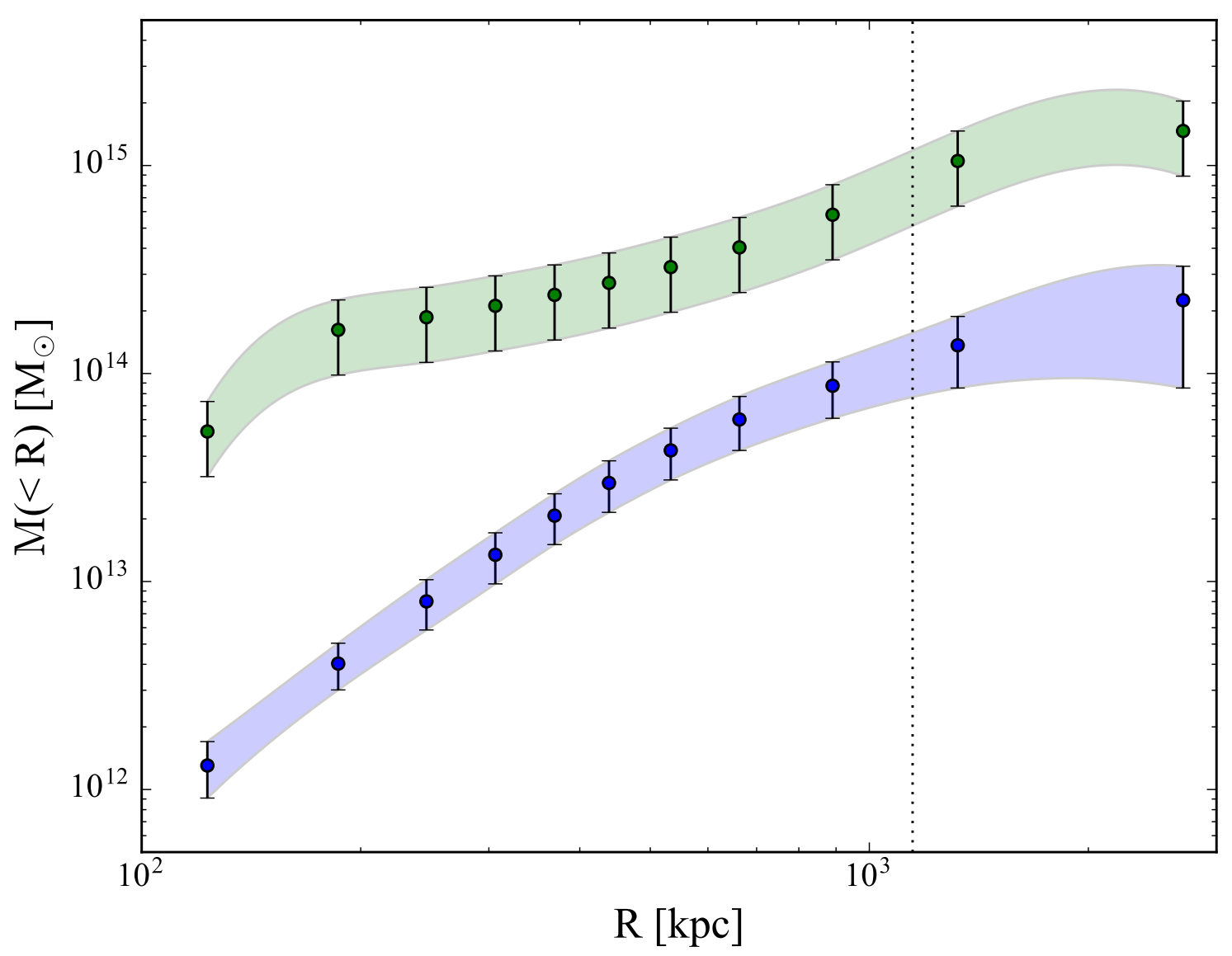

Fig. 7. Integrated mass profiles, $M(<r)$, for MACS J0025.4-1222 assuming hydrostatic equilibrium and a prolate, ellipsoidal geometry. The upper curve represents the total gravitating mass within radius, $r$, and the lower curve gives the total gas mass. The vertical dotted line indicates the $R_{500}$ radius as discussed in the text.

The gas temperature $k T$ at each radius is determined from the spectral fit directly. The electron density, $n_{\mathrm{e}}$, can be calculated directly from the normalisation of the fitted MEKAL model using

$N_{\text {MEKAL }}=1.0 \times 10^{-14} \frac{\mu}{4 \pi D_{\mathrm{A}}^{2}(1+z)^{2}} \int n_{\mathrm{e}}^{2} \mathrm{~d} V$

where $D_{\mathrm{A}}$ is the angular diameter distance at the cluster redshift $z$. For the assumed ellipsoidal geometry, the projected volume associated with each annulus can be estimated as (2/3) $S L$ where $S$ is the area of a given elliptical annulus with inner and outer semi-major axes of $r_{1}$ and $r_{2}$, respectively. The longest line-of-sight distance through the volume is denoted by $L$ and, assuming a prolate ellipsoidal geometry with major to minor axis ratio of 2 , can be expressed as $L=2\left(r_{2}^{2}-r_{1}^{1}\right)^{1 / 2}$ (Henry et al. 2004; Mahdavi et al. 2005). This approximate method of deprojection assumes that the emission along the line-of-sight is dominated by material in the volume and that the contribution from material in front and behind the volume is negligible.

With these assumptions, the resulting total gas and gravitating mass as a function of radius along the cluster semimajor is shown in Fig. 7. Based on these profiles, we estimate a total gravitating mass $M_{500}=8.44 \pm 3.16 \times 10^{14} M_{\odot}$, where $R_{500}=1.15 \mathrm{Mpc}$. This value agrees with the total mass estimate (lensing mass plus gas mass) $M_{\text {tot }}=5.65 \pm 2.2 \times 10^{14} M_{\odot}$ from Bradač et al. (2008). We note that Bradač et al. (2008) measure the lensing masses within radii of $300 \mathrm{kpc}$ of the sub-cluster BCGs, while the gas mass was measured within $500 \mathrm{kpc}$ of the ICM X-ray peak.

\subsection{Low-resolution radio imaging}

To emphasise any diffuse emission associated with the cluster merger in MACS0025, we have subtracted the clean-component model of the compact radio sources associated with the cluster, made using the high-resolution image (not presented in this paper). Subsequently, the data were re-imaged, applying a $u v$ taper of $5 \mathrm{k} \lambda$ to improve our sensitivity to large-scale radio emission. The cluster-member radio galaxies remain unresolved in both the full- and high-resolution images; consequently, flux density measurements are consistent between the full- and highresolution images. As such, we are confident that any remaining flux density associated with these cluster member RG should be negligible.

The low-resolution, source-subtracted image is presented in Fig. 8, where the synthesised beam size is $28.2 \times 23.7$ arcsec and the image rms is $270 \mu \mathrm{Jy}_{\text {beam }}^{-1}$. As can be seen in Fig. 8, we recover significant radio emission associated with the ICM on large angular scales. This represents the first detection of significant diffuse radio emission from this cluster.

Two sources of diffuse emission are present in Fig. 8, to the north-west (NW) and south-east (SE) of the cluster centre. The NW (SE) source has largest angular size of approximately 100 (90) arcsec; at the redshift of MACS0025, this corresponds to a largest linear size (LLS) of 640 (577) kpc. This is consistent with the typical size of many radio relics (e.g. Bonafede et al. 2012; de Gasperin et al. 2014) and haloes (e.g. Venturi et al. 2007, 2008; Kale et al. 2013, 2015). 


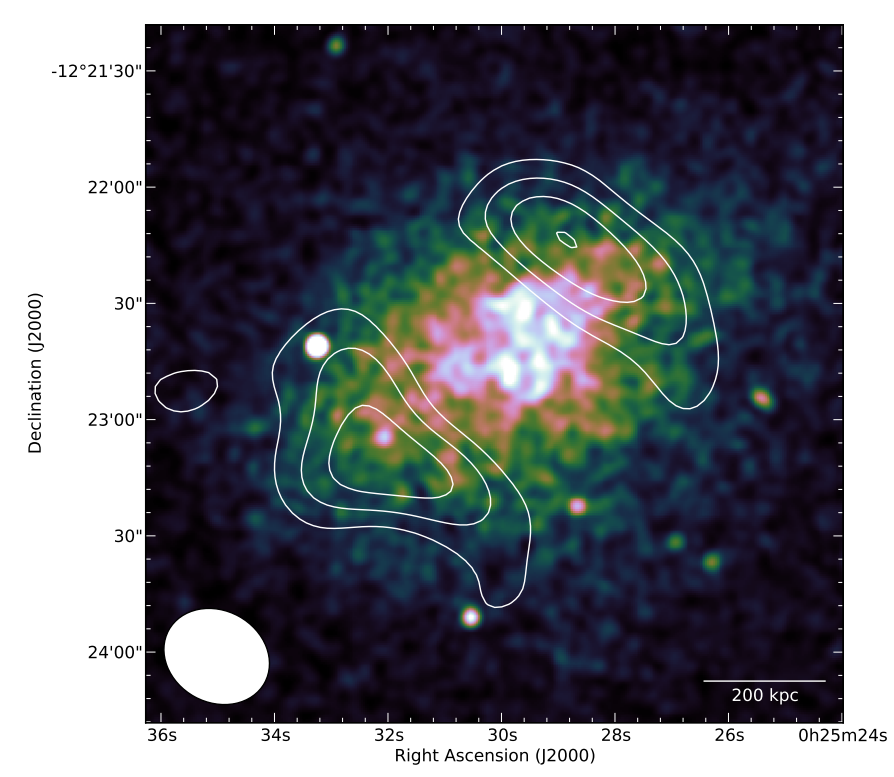

Fig. 8. Composite image of MACS0025. Colour plot is X-ray surface brightness as per Fig. 3. Contours are source-subtracted low-resolution GMRT data at $325 \mathrm{MHz}$, at levels $[5,7,9,11] \times \sigma_{\mathrm{LR}}$, where $\sigma_{\mathrm{LR}}=$ $270 \mu \mathrm{Jy}$ beam $^{-1}$. The synthesised beam in the low-resolution image is $28.2 \times 23.7$ arcsec at PA $61.2^{\circ}$, indicated by the unfilled ellipse in the lower-left corner.

These sources both exhibit an approximately arc-like morphology, are located toward the cluster periphery, and appear perpendicular to the proposed merger axis. As such, we identify this as a candidate double-relic system, and refer henceforth to these sources as the NW and SE relic. We use the fitting routine fitflux (Green 2007) to measure the flux density of the diffuse radio sources in Fig. 8. We recover an integrated flux density of $6.54 \pm 0.72(8.88 \pm 1.01) \mathrm{mJy}$ for the NW (SE) relic. The uncertainty here comes from the sum of five per cent of the integrated flux density plus the standard deviation of seven fits, plus the off-source image noise, added in quadrature.

\section{Analysis}

Given that this is the first detection of large-scale, diffuse radio emission from MACS0025, we cannot compare directly with previous work. As of January 2016, approximately 47 clusters are known to host radio relics, of which approximately 16 are known to host a pair of radio relics. In this section, we will examine how this new candidate double-relic system fits in with the known population. We include relics from both larger samples and surveys (e.g. Feretti et al. 2012; de Gasperin et al. 2014; Kale et al. 2015) as well as individual cluster studies (e.g van Weeren et al. 2012; de Gasperin et al. 2015; Shimwell et al. 2015; Cantwell et al. 2016).

\subsection{Proposed scenario: two radio relics}

From both observations of clusters, as well as simulations, it is well established that major merger events with an approximately even mass ratio should generate pairs of outward-propagating shocks, which should consequently generate a pair of radio relics (e.g. van Weeren et al. 2011, although the merger they consider has a mass ratio of approximately $2: 1$ ). Previous observations of MACS0025 have suggested that the masses of the merging clusters are approximately equal: $M \simeq 2.5 \times 10^{14} M_{\odot}$ (Bradač et al. 2008).

The morphology of the radio emission in Fig. 8 appears to be consistent with this interpretation, with both diffuse sources exhibiting approximately arc-like morphology. Additionally, both relics are detected at the periphery of the X-ray emission from the ICM, also consistent with both previous detections of relics and the suggestion that the merger event is occurring close to the plane of the sky (Bradač et al. 2008; Ma et al. 2010).

These relics have not been detected at other frequencies, so we cannot directly measure a spectral index. In the NVSS, emission is detected associated with the triplet of compact radio sources, but no emission is recovered at the $3 \sigma$ level from the location of the NW relic. Likewise, we found no positive flux below the $3 \sigma$ level in the NVSS image using fitflux; however, we can derive an upper limit from the surface brightness.

At $325 \mathrm{MHz}$, the emission from the NW relic peaks at $2.98 \mathrm{mJy}^{\text {beam }}{ }^{-1}$, which corresponds to a surface brightness of approximately $15.7 \mu \mathrm{Jy} \operatorname{arcsec}^{-2}$ given the beam size. Taking the $3 \sigma$ limit from the NVSS $\left(1.35 \mathrm{mJy}^{\text {beam }}{ }^{-1}\right.$, or $\sim 2.4 \mu \mathrm{Jy} \operatorname{arcsec}^{-2}$ ) implies an upper limit of $\alpha<-1.3$ for the spectral index of the NW relic. This is consistent with the known population; for example, the "September 2011 relic collection" of Feretti et al. (2012) has a mean spectral index $\langle\alpha\rangle=-1.3$.

We use this spectral index to scale the flux density of our relics to $1.4 \mathrm{GHz}$ and derive the radio power. Given the redshift of MACS0025 ( $z=0.5857$; Bradač et al. 2008) we find $P_{1.4 \mathrm{GHz}}=1.29 \pm 0.14 \times 10^{24} \mathrm{~W} \mathrm{~Hz}^{-1}$ for the NW relic and $P_{1.4 \mathrm{GHz}}=1.76 \pm 0.20 \times 10^{24} \mathrm{~W} \mathrm{~Hz}^{-1}$ for the SE relic, with our cosmology.

\subsubsection{Power scaling: radio power vs. X-ray luminosity}

In their review, Feretti et al. (2012) categorise cluster radio relics as "elongated" and "roundish", with the former bearing the morphological hallmarks typical of the well-known giant radio relics, and the latter exhibiting a more rounded, regular morphology. Like elongated relics, roundish relics also exhibit steep spectra, an apparent lack of an optical counterpart, as well as a high polarisation fraction. Feretti et al. (2012) include radio phoenixes under the umbrella of roundish relics. Some examples of roundish relics are those in A584b (Feretti et al. 2006), AS753 (Subrahmanyan et al. 2003) and A1664 (Govoni et al. 2001). It is worth noting that no roundish relics have been detected beyond $z \simeq 0.2$, whereas MACS0025 is at redshift $z=0.5857$ (Bradač et al. 2008). We do not include roundish relics in our analysis, as they may include a variety of different classes of source.

The X-ray luminosity of MACS0025 is $L_{\mathrm{x}}=8.8 \pm 0.2 \times$ $10^{44} \mathrm{erg} \mathrm{s}^{-1}$ (Ebeling et al. 2007). In Fig. 9 we present the $L_{\mathrm{x}} / P_{1.4 \mathrm{GHz}}$ plane for galaxy clusters hosting radio relics in the literature. Clusters hosting single radio relics are marked in blue while those hosting a pair are marked in black. From Fig. 9, the relics hosted by MACS0025 are consistent with the known population.

\subsubsection{Power scaling: radio power vs. cluster mass}

de Gasperin et al. (2014) investigate the possibility of a relation between cluster mass $\left(M_{500}\right)$ derived via the Sunyaev-Zel'dovich effect from the Planck cluster catalogue (Planck Collaboration XXIX 2014) and radio power at $1.4 \mathrm{GHz}$, finding find a strong correlation. This relationship takes the 


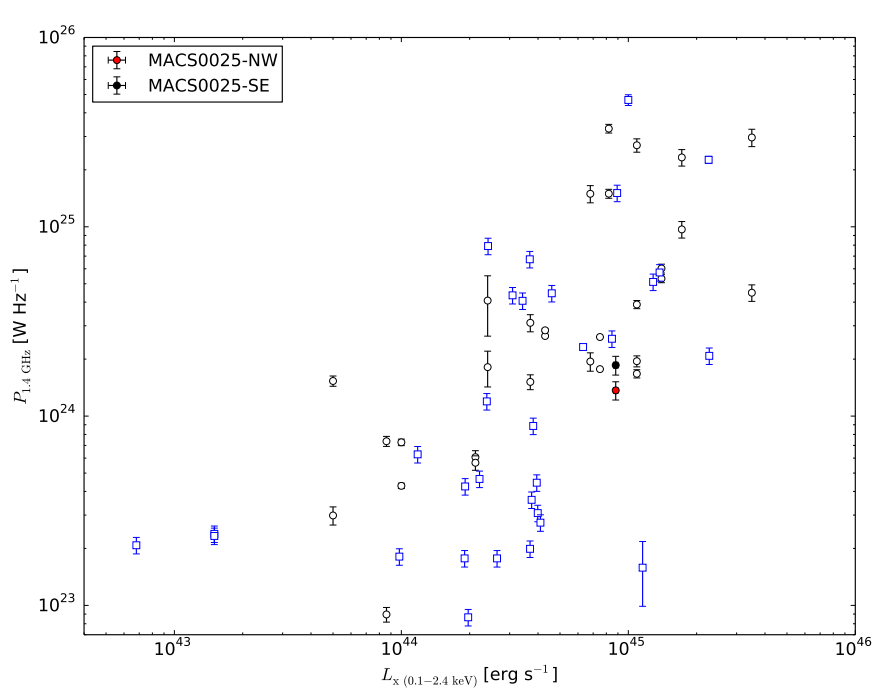

Fig. 9. Power scaling relation in the $L_{\mathrm{x}} / P_{1.4 \mathrm{GHz}}$ plane for galaxy cluster radio relics. Open circles indicate clusters hosting a pair of radio relics from the literature. Blue squares denote clusters hosting a single radio relic. Filled black (red) circles indicate the radio power and X-ray luminosity for the SE (NW) diffuse radio sources hosted by MACS0025.

form $P_{1.4 \mathrm{GHz}} \propto M_{500}^{2.83}$ for clusters hosting double-relics, and still holds (albeit with greater scatter) when clusters hosting a single relic are included.

In Fig. 10a we reproduce the data from de Gasperin et al. (2014) with the addition of a MACS0025 as well as some other clusters hosting relics that have been detected following de Gasperin et al. (2014). MACS0025 was not detected by Planck; instead we use the total gravitating mass derived from the X-ray data, $M_{500}=8.44 \pm 3.16 \times 10^{14} M_{\odot}$.

We note that Czakon et al. (2015) find a mass estimate $M_{2500}=2.38_{-0.50}^{+0.66} \times 10^{14} M_{\odot}$ for MACS0025 from the Bolocam X-ray SZ (BOXSZ; Sayers et al. 2013) sample. However, this is measured over a smaller aperture than the mass measurements used by de Gasperin et al. (2014). From Fig. 10, the relics hosted by MACS0025 are consistent with the scatter in the $M_{500} / P_{1.4 \mathrm{GHz}}$ plane for known relics, although they lie far from the relation derived by de Gasperin et al. (2014).

\subsubsection{Power scaling: radio power vs. redshift}

In Fig. 10b we present the radio power at $1.4 \mathrm{GHz}$ as a function of redshift for the known population of radio relics. The relics in MACS0025 appear to be consistent with the general trend between radio power and redshift. To date, only two other clusters beyond redshift $z=0.5$ are known to host radio relics: MACS J1149.5+2223 at $z=0.544$ (Bonafede et al. 2012) and ACT-CLJ0102-4915 at $z=0.855$ ("El Gordo"; Lindner et al. 2014). For high-redshift clusters, MACS0025 appears to be slightly low in the $P_{1.4 \mathrm{GHz}} / z$ plane, although this is likely due to selection effects and/or small number statistics.

In general, the apparent trend exhibited in the $P_{1.4 \mathrm{GHz}} / z$ plane is severely impacted by selection effects the surface brightness sensitivity of historic cluster studies has not been sufficient to detect faint relics at high redshift. The fact that the relics in MACS0025 lie slightly below the apparent correlation may suggest we are probing deeper than the majority of previous high-redshift cluster studies.

Overall, from Figs. 9 and 10 it is appears that the relics hosted by MACS0025 are consistent with established trends in

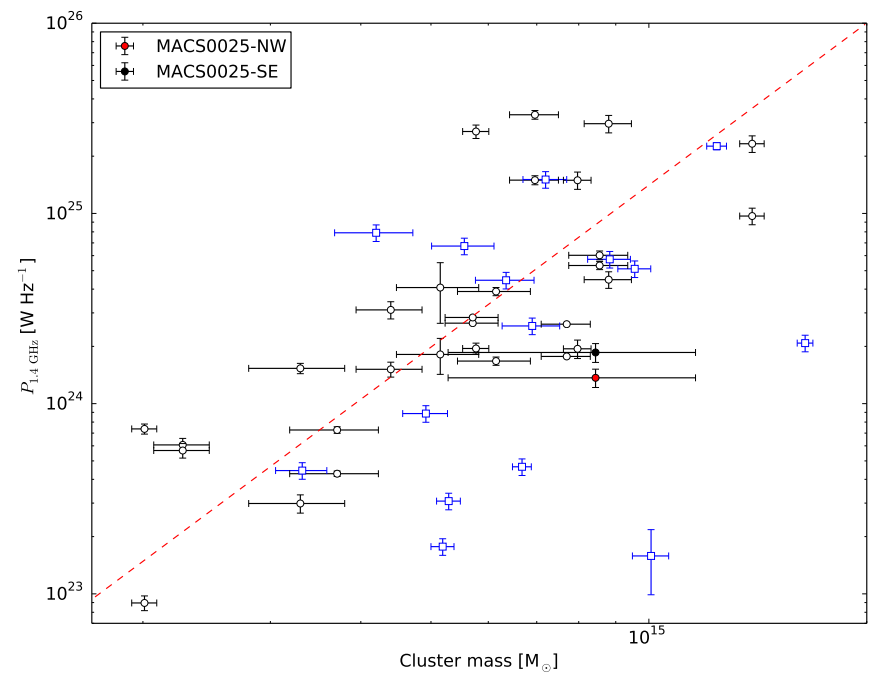

(a)

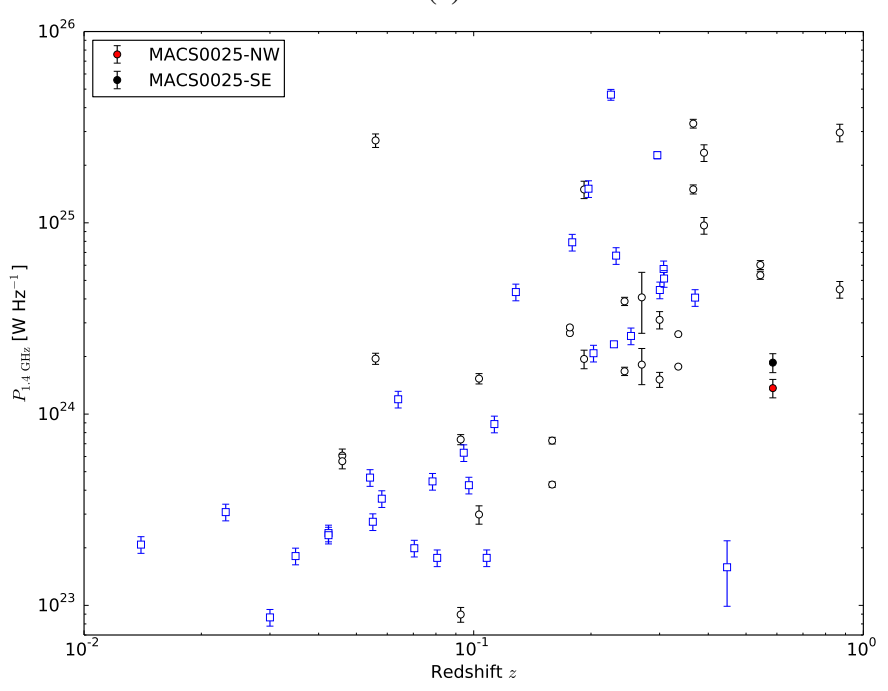

(b)

Fig. 10. Dependence of cluster relic radio power on a) cluster mass and b) redshift. Open circles indicate clusters hosting a pair of radio relics from the literature. Blue squares denote clusters hosting a single radio relic. Filled black (red) circles indicate the position of the SE (NW) diffuse radio sources hosted by MACS0025. The dashed red line in panel a) denotes the $M_{500} / P_{1.4 \mathrm{GHz}}$ relation from de Gasperin et al. (2014). Mass estimates are via the SZ-effect from the Planck cluster catalogue (Planck Collaboration XXIX 2014), or via a scaling relation based on the X-ray luminosity $\left(L_{\mathrm{x}}[0.1-2.4 \mathrm{keV}]\right.$; de Gasperin et al. 2014) for all clusters except MACS0025, where the mass is derived in Sect. 3.3.

power-scaling relations for the known population of relics. Note that the relic in MACS J2243.3-0935 (Cantwell et al. 2016) appears slightly low in all power-scaling planes; this may be due to the suggested nature as an infall relic rather than a relic generated by a merger shock, or a result of improved data reduction techniques.

\section{Discussion}

\subsection{Bulk components of the ICM}

In Fig. 11, we present a RGB optical image of MACS0025 taken with HST (from Bradač et al. 2008) overlain with false-colour 


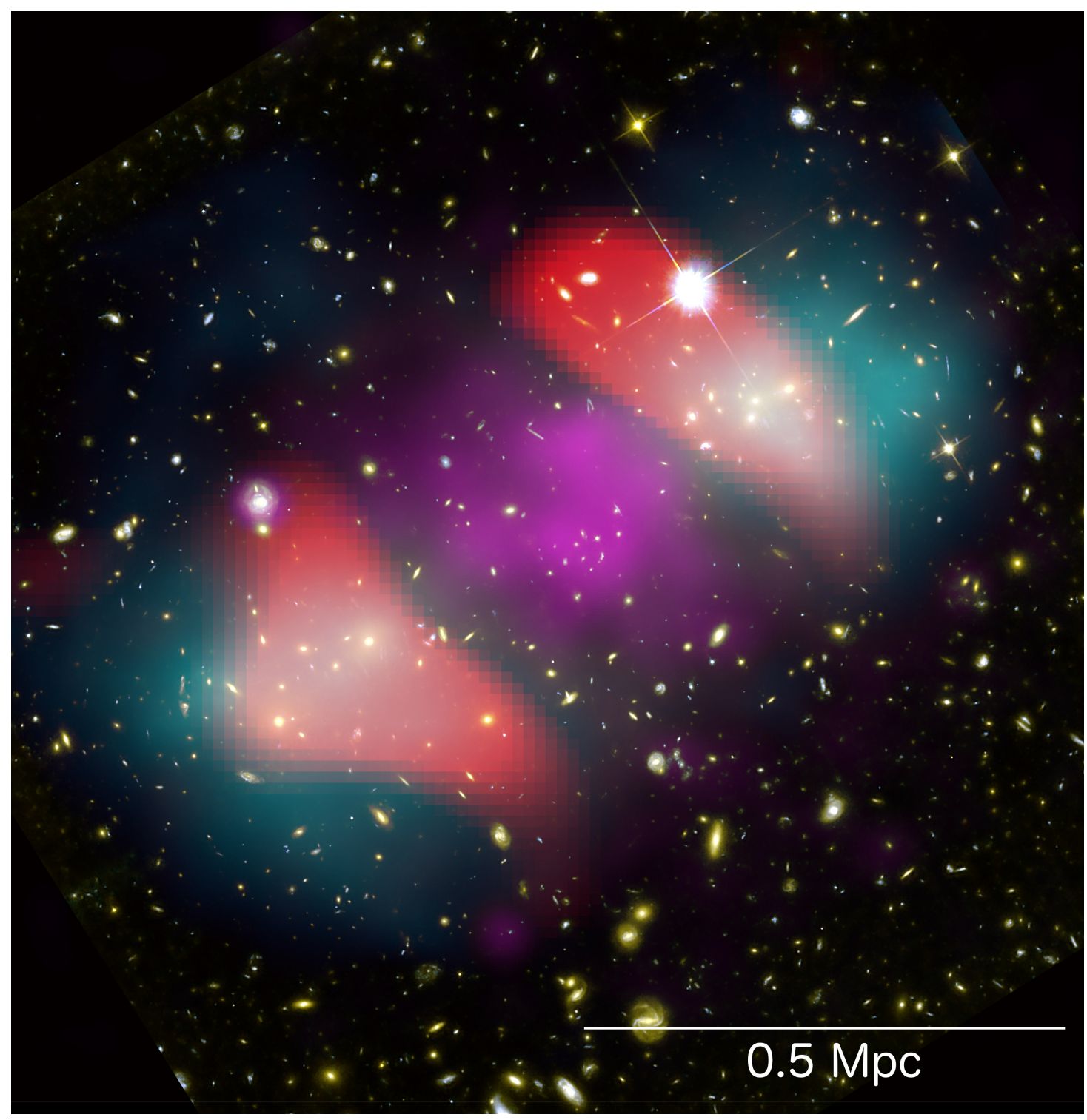

Fig. 11. False-colour image of MACS0025. Background image is a RGB optical image from HST. Cyan denotes the total mass distribution traced by lensing (dominated by the dark matter). Purple denotes the baryonic mass distribution (dominated by the ICM plasma). Red denotes the diffuse radio emission at $325 \mathrm{MHz}$. This image is in the $\mathrm{J} 2000$ coordinate system, north is up and east is to the left. X-ray and radio data are from this work whereas optical and lensing data are from Bradač et al. (2008). The scale bar denotes the angular extent corresponding to a physical distance of $0.5 \mathrm{Mpc}$ at the redshift of MACS0025.

multi-wavelength data. Figure 11 shows the relative distributions of the total mass (as traced by gravitational lensing, which is dominated by dark matter, from Bradač et al. 2008) and the baryonic mass (as traced by the X-ray surface brightness, based on the new analysis presented in this work). The lensing mass is shown in cyan and the baryonic mass in purple. Also overlain in red is the radio emission recovered by the GMRT at $325 \mathrm{MHz}$.

MACS0025 was the second galaxy cluster where significant separation was detected between the total mass and baryonic mass distributions. MACS0025 joins the Bullet cluster as part of a growing population of clusters that both host diffuse radio emission and exhibit clear separation between baryonic matter and dark matter components. Shan et al. (2010) derive the offset between the lensing and X-ray centroids for a sample of 38 galaxy clusters; in their sample are a small number of other galaxy clusters with significant offset $(\gtrsim 150 \mathrm{kpc})$ that are also known to host both a radio relic and halo: A2163 (Feretti et al. 2001) and A2744 (Govoni et al. 2001; Orrù et al. 2007).
From Bradač et al. (2008) the offsets between the lensing peaks and the baryonic matter peak (defined as the X-ray centroid) are 0.5 and 0.82 arcmin; at the redshift of MACS0025, these correspond to a physical distance of 192 and $316 \mathrm{kpc}$, respectively, for the NW (SE) sub-cluster. These offsets are comparable to the offsets for the Bullet cluster (201 kpc), A2163 (141 kpc) and A2744 (238 kpc) from Shan et al. (2010). Note that this physical size is derived using our cosmology and the measured angular offsets from Shan et al. (2010).

\subsection{Large-scale temperature structure}

In order to search for signatures of the merger in the underlying temperature structure in MACS0025, we have performed a 2D spectral analysis. As mentioned previously, the spectral extraction regions were defined using the contour binning algorithm of Sanders (2006) based on the X-ray surface brightness image and such that each region had a fixed SNR. This technique 


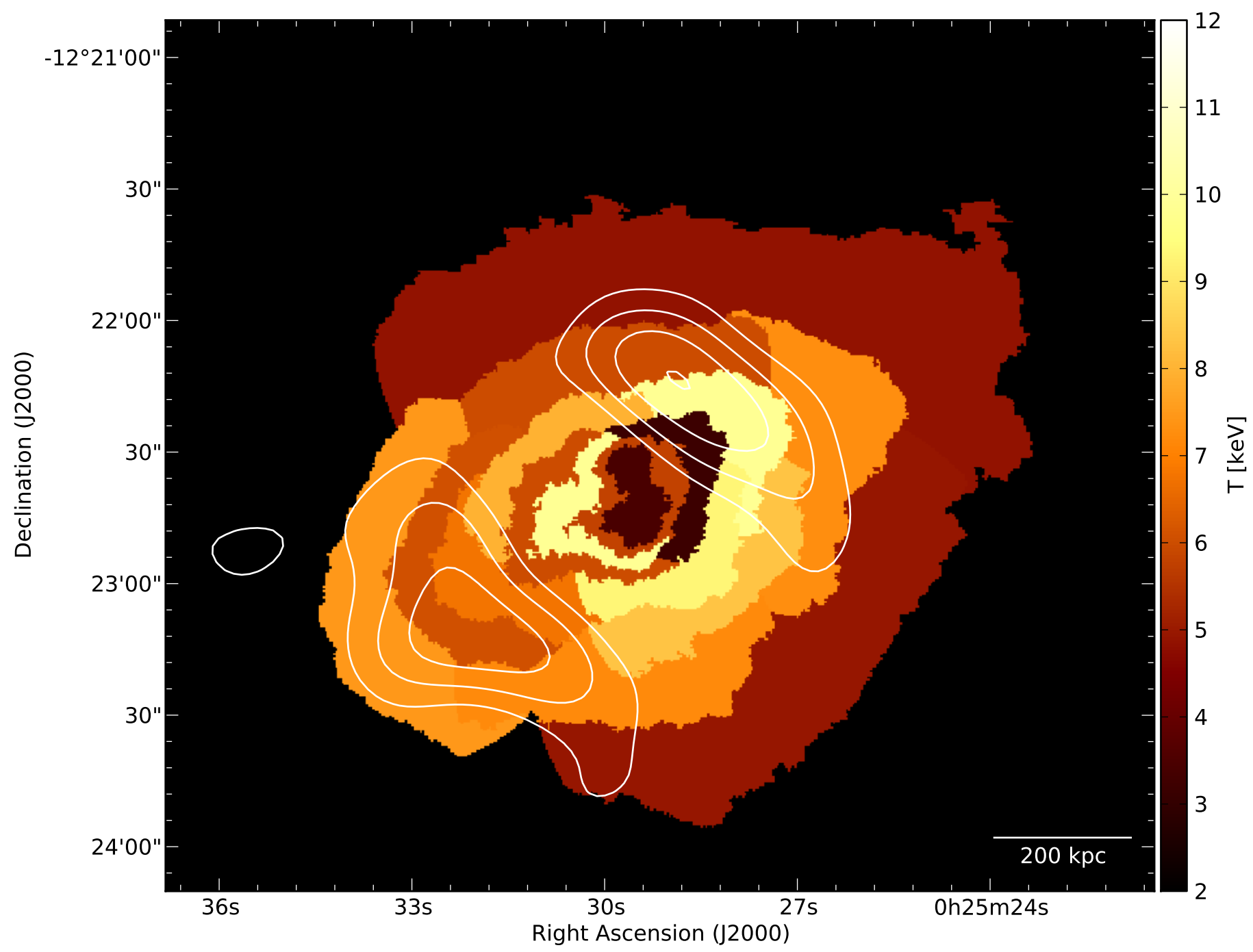

Fig. 12. X-ray temperature map of MACS0025, with low-resolution radio contours from the GMRT, as per Fig. 8.

trades off spectral accuracy against extraction region size and by extension the ability to resolve smaller-scale temperature structures. We have used a criteria of $S N R \sim 30$ which results in approximately 900 net counts per bin after background subtraction in each region. This choice represents a compromise between obtaining reasonable accuracy in the fitted temperature values while simultaneously preserving modest angular resolution.

In each of the resulting extraction regions, the standard set of source spectrum, background spectrum, effective area and response matrix files were created for each OBSID contributing to that region. These individual files were then combined into a single set of summed files for each region with the CIAO tool combine_spectra and used for all subsequent fitting. At each point in the resulting map, we have fitted a single temperature, a MEKAL thermal model (Mewe et al. 1985; Liedahl et al. 1995) and foreground Galactic absorption to the combined spectrum from that region. The Galactic absorption was fixed to a value of $N_{\mathrm{H}}=2.38 \times 10^{20} \mathrm{~cm}^{-2}$ as discussed previously in Sect. 2.2.3.

For the elemental abundance, we have derived temperature maps where the abundance was both fixed and allowed to vary spatially. The maps are qualitatively similar in both cases; however, the fitted abundances are not well-constrained in the spatially varying case and the errors on the derived temperature values are also higher. Given the higher temperature range $(\sim 6-10 \mathrm{keV})$ observed in MACS0025, the fits are relatively insensitive to the abundance. Consequently, to determine the best constraints on the temperature values, we have fixed the abundance to a value of $Z=0.23$ based on the fit to the mean spectrum discussed above in Sect. 2.2.3. The final temperature map for MACS0025 is shown in Fig. 12.

The overall temperature structure shown in Fig. 12 appears, in general, consistent with the presence of a strong merger in MACS0025. We find regions of higher temperature approximately aligned with the merger axis. We have been cautious in interpreting the maps as the contour binning algorithm combined with the lower signal-to-noise of the data can tend to enhance apparent "shell-like" substructures in the temperature. With this caveat in mind, we note that the map shown in Fig. 12 does seem to exhibit preferentially hotter gas along the merger axis presumably due to shock heating associated with the merger. However, given the limitations of the data, and the fact that the typical error in Fig. 12 is of the order of 30 to 50 per cent, this correspondence must be seen as more suggestive than definitive.

\subsection{Shocks in the ICM?}

As can be seen in Fig. 12, we have a tentative detection of regions of enhanced temperature in the ICM of MACS0025. However, as discussed, the errors are sufficiently large that Mach number 
constraints from the X-ray data will not provide tight constraints on the viability of this being a merger shock.

Additionally, the temperature structure exhibited in Fig. 12 is reminiscent of a cold front, with the cooler (unshocked) region of gas downstream of the hotter (shocked) region. However, from earlier, there is no evidence of surface brightness discontinuity that is typically associated with cold fronts (see Markevitch \& Vikhlinin 2007 for a thorough review of cold fronts in galaxy clusters).

We can estimate the Mach number based on the radio spectral index, following the formalism of Blandford \& Eichler (1987). For simple shocks,

$\mathcal{M}=\sqrt{\frac{2 \alpha_{i n j}+3}{2 \alpha_{i n j}-1}}$

where $\alpha_{\text {inj }}$ is the power-law spectral index of cosmic ray electrons $(\mathrm{CRe})$ at the shock front. Earlier, we estimated the upper limit spectral index to be $\alpha<-1.3$; hence, Eq. (5) suggests a Mach number $\mathcal{M}<1.87$. This is typical of the Mach numbers both predicted from simulations of cluster merger shocks and from observations at radio wavelengths, which suggest Mach numbers $1 \leq \mathcal{M} \leq 3$ (e.g. Feretti et al. 2012; Brunetti \& Jones 2014).

\subsection{Should MACS0025 host a radio halo?}

Whilst radio haloes and relics are strongly associated with merging clusters, the exact conditions necessary to generate these objects are far from clear. Cluster mergers deposit vast quantities of energy into the ICM and cause significant turbulence, yet not all merging clusters host a RH. Likewise, the link between merger shocks and relics is generally supported by observational evidence, but many merging clusters with prominent ICM shocks do not host relics.

Brunetti \& Jones (2014) estimate the timescale required to generate a RH following the turbulent acceleration framework, deriving $\tau_{\text {acc }} \sim 100$ Myr. Likewise, Brunetti \& Jones (2014) estimate the lifetime of radio-emitting electrons for the Coma cluster using viable magnetic field estimates, deriving $\tau_{\mathrm{CRe}} \sim$ few $\times$ 100 Myr. For comparison, Brunetti et al. (2009) estimate the lifetime of RH based on a statistical sample of approximately 19 galaxy clusters, deriving $\tau_{\mathrm{RH}} \sim 1.3 \mathrm{Gyr}$. Based on the optical properties of the cluster, Ma et al. (2010) conclude that core passage in MACS0025 occurred between 0.5 and 1 Gyr ago, suggesting that sufficient time should have elapsed to generate a $\mathrm{RH}$

From examination of double-relic systems in the literature, it appears that time since core passage $\left(\tau_{\mathrm{CP}}\right)$ and mass ratio cannot be used alone to suggest whether or not a cluster should host a RH. For example, the system CIZA J2242.8+5301 (hereafter CIZAJ2242) hosts a pair of relics but no RH (e.g. van Weeren et al. 2010). Simulations suggest that this system involves a pair of merging clusters with a mass ratio approximately 2:1, with core passage occurring around 1 Gyr ago (van Weeren et al. 2011).

In contrast, El Gordo hosts a pair of relics and a halo (Lindner et al. 2014; Botteon et al. 2016b). Weak lensing observations of El Gordo suggest that the sub-clusters also have a mass ratio of approximately $2: 1$ (Jee et al. 2014). A dynamical analysis of what is known about El Gordo is presented by $\mathrm{Ng}$ et al. (2015), who indicate two potential scenarios for the merger in El Gordo: either the system is observed shortly after first core passage $\left(\tau_{\mathrm{CP}} \sim 0.46 \mathrm{Gyr}\right)$ or the clusters are again on approach, having turned around $\left(\tau_{\mathrm{CP}} \sim 0.91 \mathrm{Gyr}\right)$.
$\mathrm{Ng}$ et al. (2015) suggest that the latter scenario is favoured if the time-averaged sub-cluster velocities exceed the shock velocity of the corresponding relic.

Both clusters also appear to have similar total masses: de Gasperin et al. (2014) find total masses $M_{\text {tot }}=7.97 \pm 0.35 \times$ $10^{14}\left(8.80 \pm 0.66 \times 10^{14}\right) M_{\odot}$ for CIZAJ2242 (El Gordo). It should be noted that these mass measurements were derived through different means; via the SZ effect for El Gordo (Planck Collaboration XXIX 2014) and via the scaling relation between X-ray luminosity and total mass (Pratt et al. 2009) for CIZAJ2242. The velocity dispersions of the sub-clusters involved in the merger events also appear to be consistent: from Jee et al. (2014) the spectroscopically-measured velocity dispersions of the NW (SE) sub-cluster is $\sigma_{\mathrm{v}}=1290 \pm$ $134(1089 \pm 200) \mathrm{km} \mathrm{s}^{-1}$ for El Gordo, compared to $\sigma_{\mathrm{v}}=$ $1160_{-90}^{+100}\left(1080_{-700}^{+100}\right) \mathrm{km} \mathrm{s}^{-1}$ (Dawson et al. 2015).

There is no evidence of a RH in MACSO025 at the $2 \sigma$ level or higher, although an upper limit to the radio power can be derived. The sensitivity of the GMRT image at $325 \mathrm{MHz}$ (Fig. 8) is $270 \mu \mathrm{Jy}$ beam $^{-1}$. Given the restoring beam FWHM $\theta=$ $28.2 \times 23.7$ arcsec, the corresponding $2 \sigma$ surface brightness limit $\mathrm{SB}_{\lim }=1.03 \mu \mathrm{Jy} \operatorname{arcsec}^{-2}$. Assuming a spherical geometry, a $1 \mathrm{Mpc}$ RH would appear to be approximately 155 arcsec in diameter at the redshift of MACS0025, given the adopted cosmology. With a typical RH spectral index $\alpha=-1.3$, this suggests a limiting radio power $P_{1.4 \mathrm{GHz}}$ lim $\simeq 4 \times 10^{24} \mathrm{~W} \mathrm{~Hz}^{-1}$. This does not place a tight constraint on the power of any potential $\mathrm{RH}$ in MACS0025.

The apparent lack of a RH in MACS0025 is not unexpected. For example, the statistical study of RH occurrence rates in clusters performed by Cuciti et al. (2015) suggests that only approximately 20 to 30 per cent of clusters with similar mass to MACS0025 host RH. Furthermore, previous surveys (e.g. Kale et al. 2015) indicate that there are many clusters in the luminosity range of MACS0025 which do not host a radio halo.

\section{Conclusions}

In this paper, we have presented the first deep radio observations of the high-redshift merging cluster MACS J0025.4-1222, performed using the GMRT at $325 \mathrm{MHz}$. The large FOV allows us to detect a population of radio galaxies that exhibit a wide range of morphologies. We also present a new analysis of all available Chandra X-ray data on this cluster.

Following subtraction of the compact radio source population, we recover two sources of diffuse emission on scales of several hundred kpc. Based on their location toward the cluster outskirts and their approximately arc-like morphology, we believe that this constitutes a new double-relic system, discovered for the first time in this cluster. We have shown that these relics are consistent with established power-scaling trends for the known relic population.

We derive an upper-limit spectral index $\alpha<-1.3$ for the NW relic; this is consistent with the spectral index of known relics. This implies a Mach number that is also consistent with what is expected from weak shocks. The X-ray data exhibit an asymmetric surface brightness profile, extended along the merger axis; we also derive a 2D temperature map of MACS J0025.4-1222, which suggests the presence of regions of enhanced temperature along the merger axis.

We tentatively identify a region co-located with the NW relic that appears to exhibit a sharp temperature discontinuity. However, its structure is perhaps more reminiscent of a cold front than a merger shock, as the cooler region of gas lies downstream of 
the hotter region. The uncertainties on the X-ray temperature are too significant to provide tight constraints on the Mach number.

Acknowledgements. We thank our anonymous referee for their careful review, which has improved the quality of our manuscript. We also thank the operators and engineers of the GMRT who made these observations possible. The GMRT is operated by the National Centre for Radio Astrophysics (NCRA) of the Tata Institute of Fundamental Research (TIFR), India. CJR wishes to thank H. Intema for many helpful conversations during the data reduction process C.J.R. gratefully acknowledges funding support from the United Kingdom Science and Technology Facilities Council (STFC). A.M.S. gratefully acknowledges support from the European Research Council under grant ERC-2012-StG 307215 LODESTONE. This work has made use of the NASA/IPAC Extragalactic Database (NED) and the NASA Astrophysics Data System (ADS). Many figures in this work have employed the "cubehelix" colour scheme (Green 2011).

\section{References}

Akamatsu, H., de Plaa, J., Kaastra, J., et al. 2012a, PASJ, 64, 49 Akamatsu, H., Takizawa, M., Nakazawa, K., et al. 2012b, PASJ, 64, 67 Alam, S., Albareti, F. D., Allende Prieto, C., et al. 2015, ApJS, 219, 12 Baars, J. W. M., Genzel, R., Pauliny-Toth, I. I. K., \& Witzel, A. 1977, A\&A, 61, 99

Bennett, A. S. 1962, MmRAS, 68, 163

Blandford, R., \& Eichler, D. 1987, Phys. Rep., 154, 1

Blasi, P., \& Colafrancesco, S. 1999, Astropart. Phys., 12, 169

Bonafede, A., Feretti, L., Giovannini, G., et al. 2009, A\&A, 503, 707

Bonafede, A., Brüggen, M., van Weeren, R., et al. 2012, MNRAS, 426, 40

Botteon, A., Gastaldello, F., Brunetti, G., \& Dallacasa, D. 2016a, MNRAS, 460, $\mathrm{L} 84$

Botteon, A., Gastaldello, F., Brunetti, G., \& Kale, R. 2016b, MNRAS, 463, 1534

Bradač, M., Clowe, D., Gonzalez, A. H., et al. 2006, ApJ, 652, 937

Bradač, M., Allen, S. W., Treu, T., et al. 2008, ApJ, 687, 959

Brunetti, G., \& Jones, T. W. 2014, Int. J. Mod. Phys. D, 23, 30007

Brunetti, G., Setti, G., Feretti, L., \& Giovannini, G. 2001, MNRAS, 320, 365

Brunetti, G., Cassano, R., Dolag, K., \& Setti, G. 2009, A\&A, 507, 661

Cantwell, T. M., Scaife, A. M. M., Oozeer, N., Wen, Z. L., \& Han, J. L. 2016, MNRAS, 458, 1803

Cavaliere, A., \& Fusco-Femiano, R. 1976, A\&A, 49, 137

Clowe, D., Bradač, M., Gonzalez, A. H., et al. 2006, ApJ, 648, L109

Cohen, A. S., Lane, W. M., Cotton, W. D., et al. 2007, AJ, 134, 1245

Condon, J. J., Cotton, W. D., Greisen, E. W., et al. 1998, AJ, 115, 1693

Conway, R. G., Kellermann, K. I., \& Long, R. J. 1963, MNRAS, 125, 261

Cuciti, V., Cassano, R., Brunetti, G., et al. 2015, A\&A, 580, A97

Cutri, R. M., Wright, E. L., Conrow, T., et al. 2014, VizieR Online Data Catalog: II/328

Czakon, N. G., Sayers, J., Mantz, A., et al. 2015, ApJ, 806, 18

Dalya, G., Frei, Z., Galgoczi, G., Raffai, P., \& de Souza, R. S. 2016, VizieR Online Data Catalog: VII/275

Dawson, W. A., Jee, M. J., Stroe, A., et al. 2015, ApJ, 805, 143

De Breuck, C., Tang, Y., de Bruyn, A. G., Röttgering, H., \& van Breugel, W. 2002, A\&A, 394, 59

de Gasperin, F., van Weeren, R. J., Brüggen, M., et al. 2014, MNRAS, 444, 3130 de Gasperin, F., Intema, H. T., van Weeren, R. J., et al. 2015, MNRAS, 453, 3483 Dennison, B. 1980, ApJ, 239, L93

Douglas, J. N., Bash, F. N., Bozyan, F. A., Torrence, G. W., \& Wolfe, C. 1996, AJ, 111, 1945

Ebeling, H., Edge, A. C., \& Henry, J. P. 2001, ApJ, 553, 668

Ebeling, H., Barrett, E., Donovan, D., et al. 2007, ApJ, 661, L33

Edge, D. O., Shakeshaft, J. R., McAdam, W. B., Baldwin, J. E., \& Archer, S. 1959, MmRAS, 68, 37

Fabricant, D., Lecar, M., \& Gorenstein, P. 1980, ApJ, 241, 552

Fanaroff, B. L., \& Riley, J. M. 1974, MNRAS, 167, 31

Feretti, L., Fusco-Femiano, R., Giovannini, G., \& Govoni, F. 2001, A\&A, 373, 106

Feretti, L., Bacchi, M., Slee, O. B., et al. 2006, MNRAS, 368, 544

Feretti, L., Giovannini, G., Govoni, F., \& Murgia, M. 2012, A\&ARv, 20, 54

Ferrari, C., Govoni, F., Schindler, S., Bykov, A. M., \& Rephaeli, Y. 2008, Space Sci. Rev., 134, 93

Finoguenov, A., Sarazin, C. L., Nakazawa, K., Wik, D. R., \& Clarke, T. E. 2010, ApJ, 715, 1143

Freeman, P., Doe, S., \& Siemiginowska, A. 2001, in Astronomical Data Analysis, eds. J.-L. Starck, \& F. D. Murtagh, Proc. SPIE, 4477, 76

Garmire, G. P., Bautz, M. W., Ford, P. G., Nousek, J. A., \& Ricker, Jr., G. R. 2003, in X-Ray and Gamma-Ray Telescopes and Instruments for Astronomy, eds. J. E. Truemper, \& H. D. Tananbaum, SPIE Conf. Ser., 4851, 28

Gopal-Krishna, \& Wiita, P. J. 2000, A\&A, 363, 507
Govoni, F., Feretti, L., Giovannini, G., et al. 2001, A\&A, 376, 803 Govoni, F., Murgia, M., Feretti, L., et al. 2005, A\&A, 430, L5 Gower, J. F. R., Scott, P. F., \& Wills, D. 1967, MmRAS, 71, 49 Green, D. A. 2007, Bull. Astron. Soc. India, 35, 77

Green, D. A. 2011, Bull. Astron. Soc. India, 39, 289 Gregory, P. C., \& Condon, J. J. 1991, ApJS, 75, 1011

Hales, S. E. G., Waldram, E. M., Rees, N., \& Warner, P. J. 1995, MNRAS, 274, 447

Henry, J. P., Finoguenov, A., \& Briel, U. G. 2004, ApJ, 615, 181

Intema, H. T., van der Tol, S., Cotton, W. D., et al. 2009, A\&A, 501, 1185

Jaffe, W. J. 1977, ApJ, 212, 1

Jee, M. J., Hughes, J. P., Menanteau, F., et al. 2014, ApJ, 785, 20

Kalberla, P. M. W., Burton, W. B., Hartmann, D., et al. 2005, A\&A, 440, 775

Kale, R., Venturi, T., Giacintucci, S., et al. 2013, A\&A, 557, A99

Kale, R., Venturi, T., Giacintucci, S., et al. 2015, A\&A, 579, A92

Kassim, N. E., Lazio, T. J. W., Erickson, W. C., et al. 2007, ApJS, 172, 686

Kellermann, K. I. 1964, ApJ, 140, 969

Kellermann, K. I., \& Pauliny-Toth, I. I. K. 1973, AJ, 78, 828

Kellermann, K. I., Pauliny-Toth, I. I. K., \& Williams, P. J. S. 1969, ApJ, 157, 1

Kerton, C. R. 2006, MNRAS, 373, 1203

Kettenis, M., van Langevelde, H. J., Reynolds, C., \& Cotton, B. 2006, in Astronomical Data Analysis Software and Systems XV, eds. C. Gabriel, C. Arviset, D. Ponz, \& S. Enrique, ASP Conf. Ser., 351, 497

Konar, C., Hardcastle, M. J., Jamrozy, M., Croston, J. H., \& Nandi, S. 2012, MNRAS, 424, 1061

Lane, W. M., Cotton, W. D., van Velzen, S., et al. 2014, MNRAS, 440, 327

Leahy, J. P., Black, A. R. S., Dennett-Thorpe, J., et al. 1997, MNRAS, 291, 20

Liedahl, D. A., Osterheld, A. L., \& Goldstein, W. H. 1995, ApJ, 438, L115

Lindner, R. R., Baker, A. J., Hughes, J. P., et al. 2014, ApJ, 786, 49

Ma, C.-J., Ebeling, H., Marshall, P., \& Schrabback, T. 2010, MNRAS, 406, 121

Macario, G., Markevitch, M., Giacintucci, S., et al. 2011, ApJ, 728, 82

Mahdavi, A., Finoguenov, A., Böhringer, H., Geller, M. J., \& Henry, J. P. 2005, ApJ, 622, 187

Markevitch, M., \& Vikhlinin, A. 2007, Phys. Rep., 443, 1

Mewe, R., Gronenschild, E. H. B. M., \& van den Oord, G. H. J. 1985, A\&AS, 62, 197

Ng, K. Y., Dawson, W. A., Wittman, D., et al. 2015, MNRAS, 453, 1531

Ogrean, G. A., Brüggen, M., van Weeren, R. J., et al. 2013, MNRAS, 433, 812

Orrù, E., Murgia, M., Feretti, L., et al. 2007, A\&A, 467, 943

Orrù, E., van Velzen, S., Pizzo, R. F., et al. 2015, A\&A, 584, A112

Paul, S., Datta, A., \& Intema, H. T. 2014, ArXiv e-prints [arXiv: 1412. 0285]

Pauliny-Toth, I. I. K., Wade, C. M., \& Heeschen, D. S. 1966, ApJS, 13, 65

Petrosian, V. 2001, ApJ, 557, 560

Pizzo, R. F., de Bruyn, A. G., Bernardi, G., \& Brentjens, M. A. 2011, A\&A, 525, A104

Planck Collaboration XXIX. 2014, A\&A, 571, A29

Pratt, G. W., Croston, J. H., Arnaud, M., \& Böhringer, H. 2009, A\&A, 498, 361

Rengelink, R. B., Tang, Y., de Bruyn, A. G., et al. 1997, A\&AS, 124, 259

Roger, R. S., Costain, C. H., \& Bridle, A. H. 1973, AJ, 78, 1030

Roy, J., Gupta, Y., Pen, U.-L., et al. 2010, Exp. Astron., 28, 25

Saikia, D. J., \& Jamrozy, M. 2009, Bull. Astron. Soc. India, 37, 63

Sanders, J. S. 2006, MNRAS, 371, 829

Sarazin, C. L., \& Bahcall, J. N. 1977, ApJS, 34, 451

Sayers, J., Czakon, N. G., Mantz, A., et al. 2013, ApJ, 768, 177

Scaife, A. M. M., \& Heald, G. H. 2012, MNRAS, 423, L30

Schilizzi, R. T., Tian, W. W., Conway, J. E., et al. 2001, A\&A, 368, 398

Schoenmakers, A. P., de Bruyn, A. G., Röttgering, H. J. A., van der Laan, H., \& Kaiser, C. R. 2000, MNRAS, 315, 371

Shan, H., Qin, B., Fort, B., et al. 2010, MNRAS, 406, 1134

Shimwell, T. W., Markevitch, M., Brown, S., et al. 2015, MNRAS, 449, 1486

Subrahmanyan, R., Beasley, A. J., Goss, W. M., Golap, K., \& Hunstead, R. W. 2003, AJ, 125, 1095

van Haarlem, M. P., Wise, M. W., Gunst, A. W., et al. 2013, A\&A, 556, A2

van Weeren, R. J., Röttgering, H. J. A., Brüggen, M., \& Cohen, A. 2009, A\&A, 505,991

van Weeren, R. J., Röttgering, H. J. A., Brüggen, M., \& Hoeft, M. 2010, Science, 330,347

van Weeren, R. J., Brüggen, M., Röttgering, H. J. A., \& Hoeft, M. 2011, MNRAS, 418, 230

van Weeren, R. J., Röttgering, H. J. A., Intema, H. T., et al. 2012, A\&A, 546, A124

Venturi, T., Giacintucci, S., Brunetti, G., et al. 2007, A\&A, 463, 937

Venturi, T., Giacintucci, S., Dallacasa, D., et al. 2008, A\&A, 484, 327

Vikhlinin, A., Markevitch, M., Murray, S. S., et al. 2005, ApJ, 628, 655

White, R. L., \& Becker, R. H. 1992, ApJS, 79, 331

Wright, E. L. 2006, PASP, 118, 1711

Wright, A. E., Griffith, M. R., Burke, B. F., \& Ekers, R. D. 1994, ApJS, 91, 111

Zhang, X., Zheng, Y., Chen, H., et al. 1997, A\&AS, 121, 59 


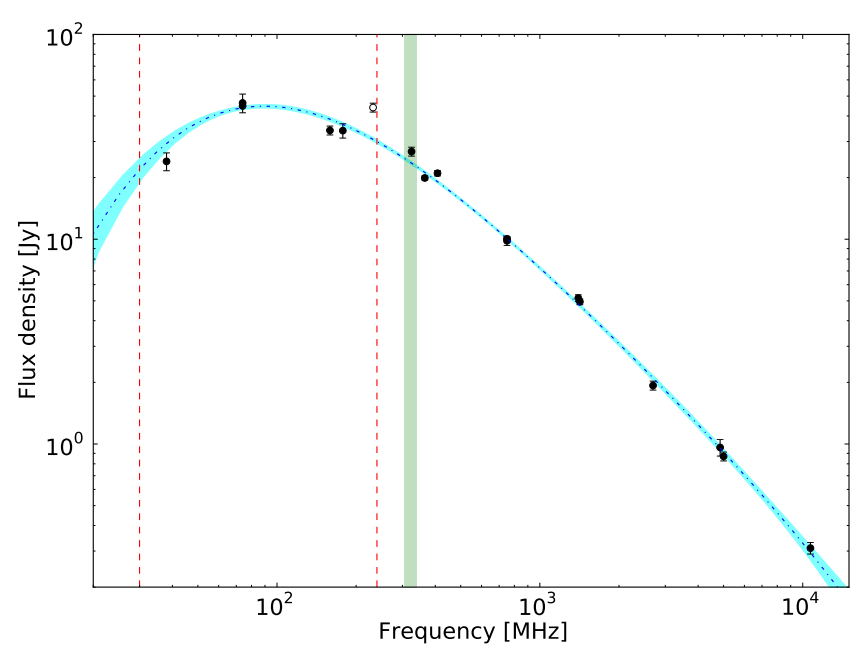

Fig. A.1. Integrated flux density of 3C 468.1 as a function of frequency. Dot-dashed line marks fourth-order polynomial fit to the data. Empty circle indicates measurement from the Miyun survey at $232 \mathrm{MHz}$ which has been excluded from the fit. Shaded green region marks the frequency range covered by the GMRT observations in this work; shaded blue region marks $1 \sigma$ uncertainty region on the model. Vertical dashed lines mark the frequency bounds of LOFAR.

\section{Appendix A: An extension to the Scaife and Heald flux scale: $3 C 468.1$}

The radio source $3 \mathrm{C} 468.1$ is available as a primary calibrator for GMRT observations, although it is perhaps the least preferred primary calibrator. No other calibrator source was available for the initial flux calibrator scan for our observations, however. The SPAM calibration routine makes use of the SH12 flux density scale, which does not include 3C 468.1. To avoid potential issues associated with using different flux density scales, therefore, we follow the method adopted by SH12 to fit the available flux density measurements for 3C 468.1 and add it to the SH12 flux density scale.

Flux density measurements exist between $38 \mathrm{MHz}$ and $10 \mathrm{GHz}$ for 3C 468.1, with observations using a range of different flux density scales. Following SH12 we bring these measurements onto the flux scale of Roger et al. (1973; hereafter RCB). We present these measurements, along with the correction factor required to bring them onto the RCB scale, in Table A.1. All flux density measurements on the Baars scale were adjusted by interpolation of the values listed in Table 7 of Baars et al. (1977; hereafter B77).

For the Texas radio survey at $365 \mathrm{MHz}$, Douglas et al. (1996) find their flux density measurements to be consistent with a factor of 96 per cent of the B77 flux scale. Below $325 \mathrm{MHz}$, the conversion process is a little more complex. For measurements on the Kellermann et al. (1969; hereafter KPW) scale at $178 \mathrm{MHz}$, RCB give a conversion factor of 1.09. For the measurement from Gower et al. (1967) on the CKL scale (Conway et al. 1963) Table 7 of B77 was used to convert to the RCB scale and then onto the SH12 scale. For the measurements at $74 \mathrm{MHz}$ using the VLA (Cohen et al. 2007 and Kassim et al. 2007) the B77 scale was used. Lane et al. (2014) find that a mean conversion factor of 1.1 is required to bring flux density measurements into line with the SH12 flux scale.

We present the flux density measurements as a function of frequency in Fig. A.1. We exclude the $232 \mathrm{MHz}$ flux density from the Miyun survey from the fitting partly due to a large discrepancy between the integrated flux density (43.98 Jy) and the
Table A.1. Integrated flux density measurements for 3C 468.1 from the literature, along with the flux density scale used in the original reference, and the "correction" factor required to bring the measurement onto the SH12 scale.

\begin{tabular}{|c|c|c|c|c|}
\hline $\begin{array}{c}\text { Frequency } \\
{[\mathrm{MHz}]}\end{array}$ & $\begin{array}{l}S_{\text {int }} \\
{[\mathrm{Jy}]}\end{array}$ & Flux scale & Factor & Catalogue reference \\
\hline 10700 & $0.31 \pm 0.02$ & KPW & - & KPT \\
\hline 5000 & $0.87 \pm 0.04^{a}$ & KPW & - & KPW \\
\hline 4850 & $0.96 \pm 0.09$ & B77 & 1.007 & GC91 \\
\hline 2695 & $1.93 \pm 0.10^{a}$ & KPW & - & KPW \\
\hline 1420 & $5.78 \pm 0.17$ & B77 & 0.972 & K06 \\
\hline 1400 & $5.00 \pm 0.25^{a}$ & KPW & - & KPW \\
\hline 1400 & $4.95 \pm 0.15$ & B77 & 0.972 & C98 \\
\hline 1400 & $4.89 \pm 0.24$ & B77 & 0.972 & WB91 \\
\hline 1400 & $5.16 \pm 0.13$ & K64 & 1.009 & PWH \\
\hline 750 & $9.70 \pm 0.49^{a}$ & KPW & - & KPW \\
\hline 750 & $10.06 \pm 0.15$ & K64 & 1.012 & PWH \\
\hline 408 & $21.50 \pm 0.64$ & $\mathrm{~B} 77 / \mathrm{RCB}$ & - & K06 \\
\hline 365 & $22.13 \pm 0.51$ & $\mathrm{TXS}^{c}$ & 0.977 & D96 \\
\hline 325 & $26.80 \pm 1.34$ & WENSS $^{d}$ & 0.90 & R97 \\
\hline 232 & 43.98 & $\mathrm{RCB}$ & - & Z97 \\
\hline 178 & $30.00 \pm 3.00^{b}$ & KPW & 1.09 & KPW \\
\hline 178 & $30.50 \pm 2.40^{e}$ & CKL & $1.15^{f}$ & G67 \\
\hline 159 & $34.00 \pm 3.40^{g}$ & $\mathrm{RCB}$ & - & B62 \\
\hline 74 & $40.70 \pm 0.66$ & B77 & 1.1 & K07 \\
\hline 74 & $42.09 \pm 4.41$ & B77 & 1.1 & $\mathrm{C} 07$ \\
\hline 38 & $24.60 \pm 2.46^{b}$ & $\mathrm{RCB}$ & - & H95 \\
\hline
\end{tabular}

Notes. ${ }^{(a)}$ The uncertainty is quoted as 5 per cent of the integrated flux density. ${ }^{(b)}$ The uncertainty is quoted as 10 per cent of the integrated flux density. ${ }^{(c)}$ Sources in the Texas radio survey were found to have flux densities that were consistent with a factor $0.9607 \times$ B77. See Douglas et al. (1996). ${ }^{(d)}$ The WENSS flux scale is complex. An overall correction factor of 0.9 was applied to bring the flux density onto the B77 scale, then to the RCB73 scale. See Scaife \& Heald (2012) for details. ${ }^{(e)}$ The uncertainty is quoted as 8 per cent of the integrated flux density. ${ }^{(f)}$ This conversion factor was derived using the factors in Table 7 of B77 to convert to the KPW scale and then onto SH12. ${ }^{(g)}$ An uncertainty of 10 per cent has been assumed.

References. B62: Bennett (1962); B77: Baars et al. (1977); C07: Cohen et al. (2007); C98: Condon et al. (1998); CKL: Conway et al. (1963); D96: Douglas et al. (1996); G67: Gower et al. (1967); GC91: Gregory \& Condon (1991); H95: Hales et al. (1995); K06: Kerton (2006); K07: Kassim et al. (2007); K64: Kellermann (1964); KPT: Kellermann \& Pauliny-Toth (1973); KPW: Kellermann et al. (1969); PWH: Pauliny-Toth et al. (1966); R97: Rengelink et al. (1997); RCB: Roger et al. (1973); SH12: Scaife \& Heald (2012); WB91: White \& Becker (1992); Z97: Zhang et al. (1997).

peak flux density (38.77 Jy) and partly due to the large offset with respect to the other flux densities from the literature (see Fig. A.1). Following SH12, we attempt to fit a polynomial model in linear frequency space in order to retain Gaussian noise characteristics. As such, the functional form we attempt to fit is as follows:

$S[\mathrm{mJy}]=\mathrm{A}_{0} \prod_{\mathrm{i}=1}^{\mathrm{N}} 10^{\mathrm{A}_{\mathrm{i}} \log ^{\mathrm{i}}[v / 150 \mathrm{MHz}]}$.

The best-fit parameters for linear, second-, third- and fourthorder polynomial functions are listed in Table A.2 (and presented in Fig. A.2) along with the associated uncertainties and the reduced chi-squared $\left(\chi_{\text {red }}^{2}\right)$ which is used to evaluate the goodness of fit. From visual inspection it is clear that the linear and secondorder models are a poor fit to the data; it also appears that a 

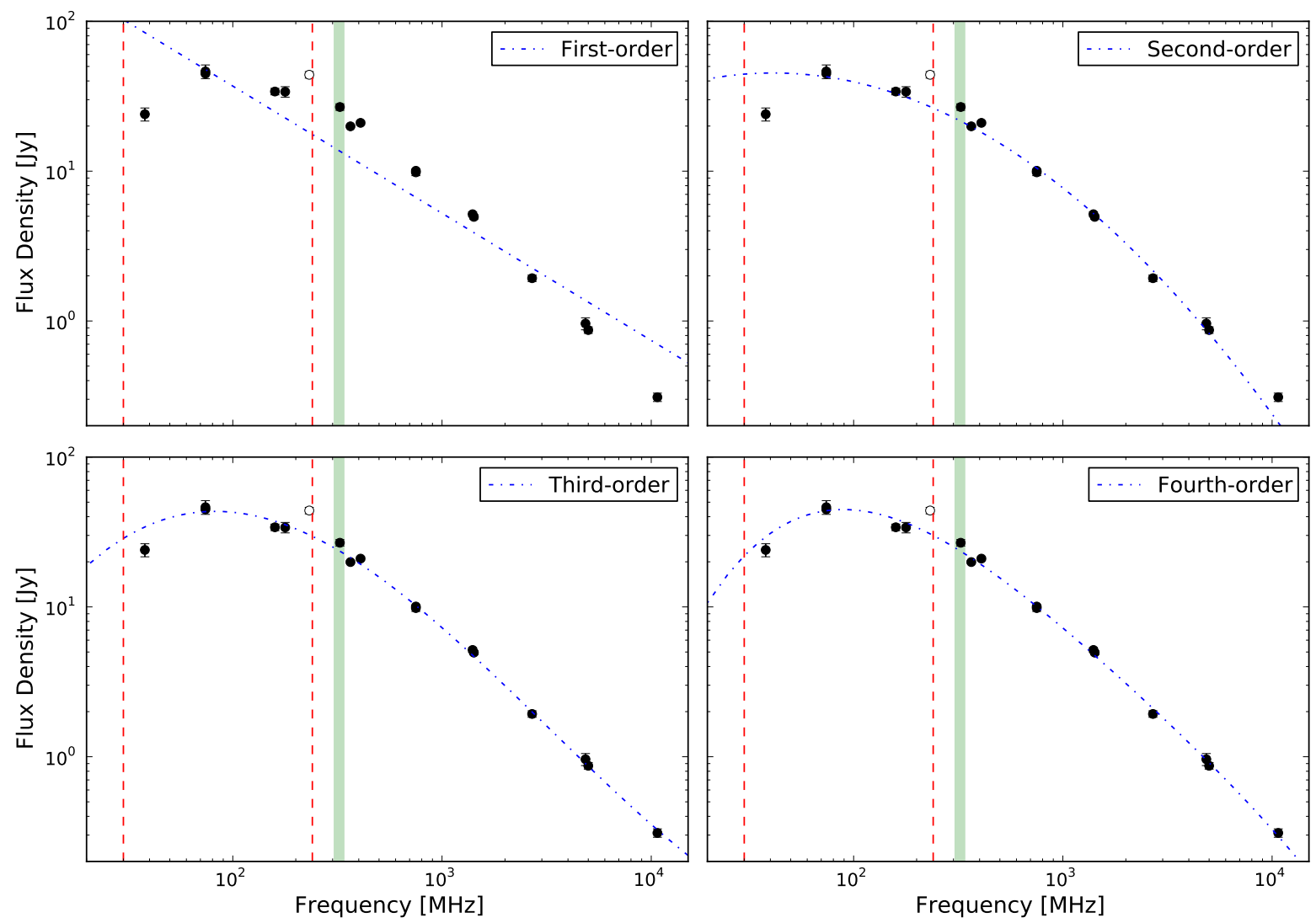

Fig. A.2. Polynomial spectral index models fitted to 3C 468.1, showing linear, second-, third- and fourth-order polynomial models from left to right. Empty circle indicates measurement from the Miyun survey at $232 \mathrm{MHz}$ which has been excluded from the fit. Shaded green region marks the frequency range covered by the GMRT observations in this work; vertical dashed lines mark the frequency bounds of LOFAR.

Table A.2. Polynomial spectral index model parameters for 3C 468.1.

\begin{tabular}{c|rrrr}
\hline \hline Order & 1 & 2 & 3 & 4 \\
\hline$A_{0}$ & $26.977 \pm 3.138$ & $34.432 \pm 1.263$ & $38.751 \pm 1.252$ & $40.093 \pm 1.530$ \\
$A_{1}$ & $-0.840 \pm 0.054$ & $-0.440 \pm 0.035$ & $-0.392 \pm 0.026$ & $-0.420 \pm 0.030$ \\
$A_{2}$ & - & $-0.415 \pm 0.036$ & $-0.715 \pm 0.068$ & $-0.830 \pm 0.104$ \\
$A_{3}$ & - & - & $0.174 \pm 0.034$ & $0.389 \pm 0.144$ \\
$A_{4}$ & - & - & - & $-0.084 \pm 0.053$ \\
$\chi_{\text {red }}^{2}$ & 5.89 & 0.96 & 0.37 & 0.24 \\
\hline
\end{tabular}

fourth-order polynomial describes the data marginally better at the low end of the frequency range.

In the case of $3 \mathrm{C} 468.1$, selection of the best-fit model is heavily influenced by the confidence in the single data point at $38 \mathrm{MHz}$; this flux density measurement is from the revised 3C catalogue (3CR; Hales et al. 1995). Based on this, and the $\chi_{\text {red }}^{2}$ values, we select a fourth-order polynomial as the "bestfit" model for the spectral index behaviour of 3C 468.1. In Fig. A.1 we present the fourth-order fit to the data shown in Table A.1. The uncertainty in the model was derived using a Monte-Carlo simulation of the parameter space over 1000 iterations; in Fig. A.1 the $1 \sigma$ uncertainty is indicated by the shaded cyan region.

\section{Appendix B: Radio environment of MACS0025}

In this Appendix we present postage stamp images of the extended radio sources identified in Fig. 2. For each $R G$, we again measured the flux density recovered by the GMRT using fitflux (Green 2007). These measurements are presented in Table B.1.
Table B.1. Measured parameters for extended radio sources in the MACS0025 field, as seen by the GMRT at $325 \mathrm{MHz}$.

\begin{tabular}{ccccc}
\hline \hline Source & $\begin{array}{c}S_{\text {int }} \\
{[\mathrm{Jy}]}\end{array}$ & $\begin{array}{c}\text { LAS } \\
{[\operatorname{arcsec}]}\end{array}$ & $z$ & $\begin{array}{c}\text { LLS } \\
{[\mathrm{kpc}]}\end{array}$ \\
\hline RG1 & $0.925 \pm 0.046$ & 83 & - & - \\
RG2 & $0.564 \pm 0.028$ & 282 & 0.153 & 721 \\
RG3 & $0.059 \pm 0.003$ & 130 & 0.162 & 347 \\
RG4 & $0.109 \pm 0.006$ & 130 & $\sim 0.129$ & $\sim 287$ \\
RG5 & $0.097 \pm 0.005$ & 78 & 0.219 & 266 \\
RG6 & $0.511 \pm 0.026$ & 170 & 0.210 & 561 \\
RG7 & $1.483 \pm 0.075$ & 86 & 0.382 & 419 \\
\hline
\end{tabular}

Notes. Flux densities were measured using fitflux (Green 2007). Largest angular sizes are measured directly from the postage stamp images, and converted to a largest linear size for sources with identified potential host galaxies. Redshift references are listed in the text.

Postage stamp images of these RG are presented in Fig. B.1, where contours start at $5 \sigma$ and scale by a factor of two. The 

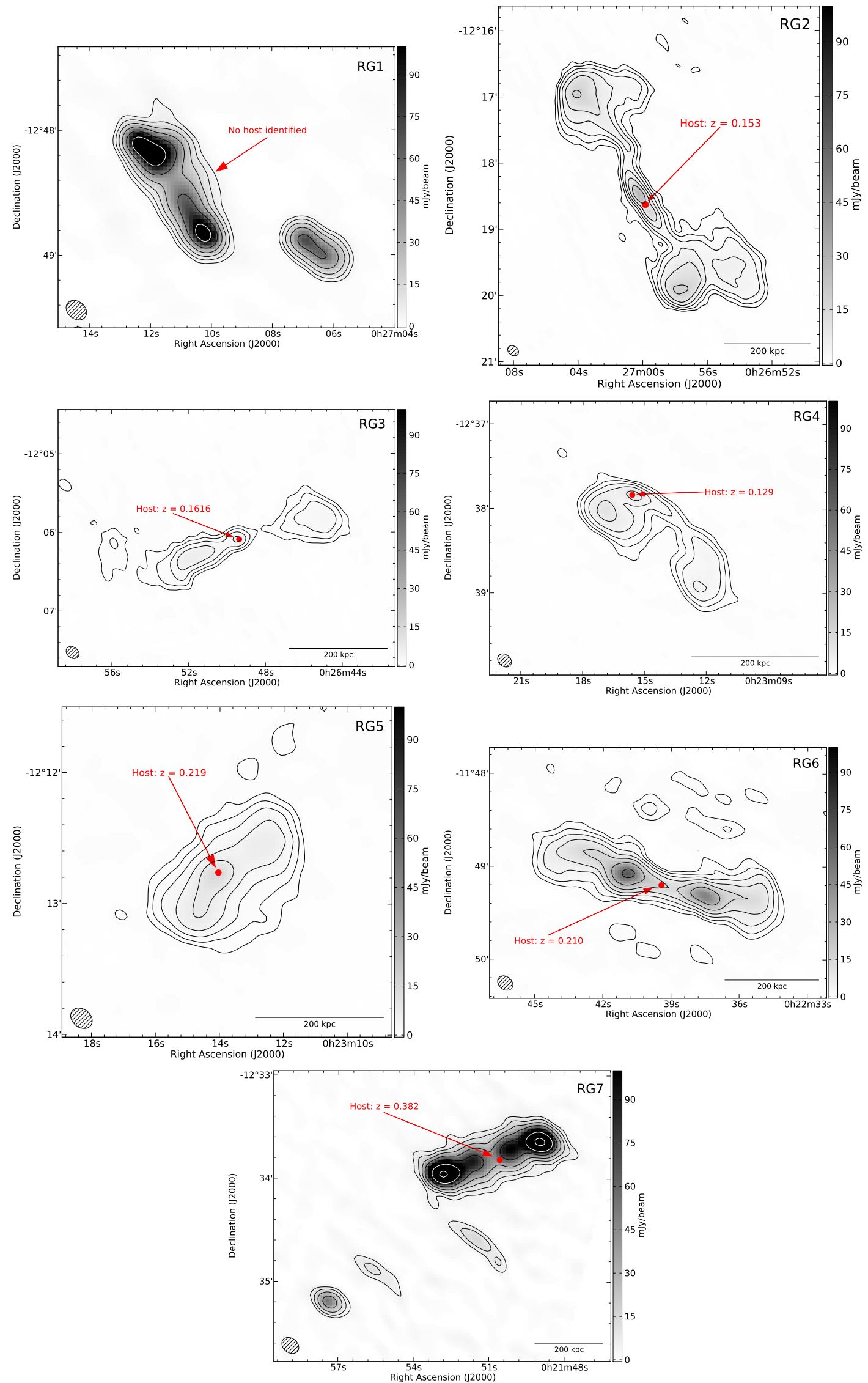

Fig. B.1. Postage stamp images of resolved sources in the GMRT field of view at $325 \mathrm{MHz}$. Contours start at $5 \sigma$ and scale by a factor of two, where $\sigma=697 / 102 / 89 / 94 / 93 / 175 / 875 \mu \mathrm{Jy}_{\text {beam }}{ }^{-1}$ from top-left. Resolution is $10.1 \times 8.3 \mathrm{arcsec}$, indicated by the hatched ellipse in the lowerleft corner. Colour scales saturate at $100 \mathrm{mJy}_{\text {beam }}{ }^{-1}$. Filled circles indicate the position of candidate host sources, where available. The scale bar denotes $200 \mathrm{kpc}$ at the redshift of sources whose proposed hosts have measured $z$. 
local noise for each postage stamp is noted in the caption of Fig. B.1. We have cross-referenced these sources with the literature to identify potential optical host galaxies and we will discuss them in this Appendix.

Approximately half of this field is covered by the 12th Data Release from the Sloan Digital Sky Survey (SDSS DR12; Alam et al. 2015) from which we identify optical host galaxies with photometric redshifts for four RG. None of these objects have spectroscopic redshifts. In Fig. B.1 filled circles indicate the location of any potential hosts.

\section{B.1. RG1}

This source appears to be a double-lobed radio source, with an approximate angular extent of 83 arcsec. We do not identify any candidate host galaxy for this radio source from the literature. The nearest source catalogued is AllWISE J002711.53-124819.1 (Cutri et al. 2014) although this appears to be co-located with the northern radio lobe, and is perhaps unlikely to host the observed radio emission.

\section{B.2. RG2: a large-angular-size radio galaxy}

The potential host for this source is identified as 2MASX J00265983-1218378 (RA 00 $26^{\mathrm{m}} 59.8^{\mathrm{s}}$, Dec $-12^{\circ} 18^{\prime} 38^{\prime \prime}$ ) at a redshift $z=0.153$. From Fig. B.1, this host appears to be in a suitable position to host the radio emission. The angular distance between the hotspots of the radio lobes is of the order of 4.6 arcmin; with the cosmology we adopt in this work, this suggests a physical extent of the order of $705 \mathrm{kpc}$; slightly smaller than giant radio galaxies.

This source is well-resolved, with a marked difference in morphology between the northern and southern radio lobes. The northern lobe appears reasonably symmetric, whereas the southern lobe exhibits a highly asymmetric radio surface brightness. On the one hand, the asymmetry may indicate differences in the density of the local environment, which restricts the expansion of part of the southern lobe. On the other hand, this fainter region may be the remains of a radio lobe emitted during historic AGN activity, whereas the brighter region may be fresher plasma from a more recent phase of activity. The northern jet also exhibits a curious "kink", which may be distorted due to the density profile of the local environment, or variation in the magnetic field orientation along the jet.

\section{B.3. RG3: a hybrid-morphology radio source}

RG3 is a complex case, exhibiting characteristics of both Fanaroff-Riley Class I (FR-I) and Class II (FR-II) radio galaxies (Fanaroff \& Riley 1974). The eastern lobe appears relatively bright and amorphous, with no strong evidence of a jet (FR-II). In contrast, the radio emission to the west of the core appears to be more confined for a greater distance out from the central engine. Additionally, the radio emission appears to be connected to a bright central core by a jet. Finally, further to the west there is patchy emission that, if associated with this source, might suggest bending of the jet.

Sources with such mixed morphology are known as hybridmorphology radio sources (HYMORS; Gopal-Krishna \& Wiita 2000). A potential optical host is identified from the GLADE catalogue $^{2}$ (Dalya et al. 2016). This source has a redshift $z=$ 0.1617; at this redshift, the angular extent of this source (130 arcsec) corresponds to a physical extent of $347 \mathrm{kpc}$.

\section{B.4. RG4}

This source exhibits a curious morphology, reminiscent of the radio lobes of wide-angle-tail (WAT) RG seen in many galaxy clusters. However, no host is identified at a location consistent with this interpretation. Instead, we find a galaxy coincident with one of the "hotspots" in the NE region; SDSS J002315.60-123750.2 $(z=0.129)$, and no obvious host for the SW emission.

A number of other galaxies are identified by the SDSS in this region, although they are distributed across a broad enough redshift range $(z=0.108-0.414)$ that we cannot suggest that they form a group. If the radio emission is associated with SDSS J002315.60-123750.2, its angular extent (130 arcsec) corresponds to a physical distance of $287 \mathrm{kpc}$. The location of the host would then suggest that we are viewing this object in projection.

\section{B.5. RG5}

We identify a host galaxy for this object, SDSS J002314.06-121245.7 $(z=0.219)$. The angular extent of this source $(78$ arcsec) therefore corresponds to a physical extent of $266 \mathrm{kpc}$ given our cosmology. At radio wavelengths, this source is also listed in the NVSS catalogue and $352 \mathrm{MHz}$ WISH catalogue (De Breuck et al. 2002) under the identifiers NVSS 002313-121246 and WNB 0020.6-1229, respectively.

\section{B.6. RG6: a candidate double-double radio galaxy}

From the SDSS, we identify the host galaxy as SDSS J002239.44-114912.0 $(z=0.210)$. At this redshift, the angular extent of this source $(170 \mathrm{arcsec})$ corresponds to a physical extent of $561 \mathrm{kpc}$.

When viewed in concert with the host location, the source morphology suggests that RG6 may be a double-double radio galaxy (DDRG). These sources exhibit themselves as pairs of double-lobed radio sources, where the lobes are aligned along the same axis and possess a common radio core (Schoenmakers et al. 2000). A number of other examples of DDRG exist in the literature (see for example Leahy et al. 1997; Schoenmakers et al. 2000; Schilizzi et al. 2001; Saikia \& Jamrozy 2009; Konar et al. 2012; Orrù et al. 2015). DDRG are believed to arise as a result of recurrent AGN activity.

This source has also been identified in a number of radio surveys, including the $4.85 \mathrm{GHz}$ Parkes-MIT-NRAO survey (PMN; Wright et al. 1994) under the identifier PMN J0022-1148, as well as the VLSS, WISH, and NVSS.

\section{B.7. RG7}

The morphology of this source is reminiscent of a classic double-lobed RG, with a pair of bright diametrically-opposed hotspots, and extended emission trails back toward the location of a central engine. This source has been catalogued by previous surveys between $74 \mathrm{MHz}$ (VLSS) and $4.85 \mathrm{GHz}$ (PMN). We identify a potential host galaxy for this source SDSS J002150.58-123349.0. This source has a photometric redshift $z=0.382$; at this redshift, the angular size of this source (83 arcsec) corresponds to a physical extent of $419 \mathrm{kpc}$.

\footnotetext{
2 Available at http://aquarius.elte.hu/glade/
} 